ALEA, Lat. Am. J. Probab. Math. Stat. 14, 63-92 (2017)

DOI: $10.30757 /$ ALEA.v14-05

\title{
Limiting behavior for a general class of voter models with confidence threshold
}

\author{
Nicolas Lanchier and Stylianos Scarlatos \\ School of Mathematical and Statistical Sciences, Arizona State University, Tempe, Ari- \\ zona, AZ 85287-1804, USA. \\ E-mail address: nlanchie@asu.edu \\ Medical Physics Laboratory, Democritus University of Thrace 68100, Alexandroupolis, \\ Greece. \\ E-mail address: stylian.scarlatos@gmail.com
}

\begin{abstract}
This article is concerned with a general class of stochastic spatial models for the dynamics of opinions. Like in the one-dimensional voter model, individuals are located on the integers and update their opinion at a constant rate based on the opinion of their neighbors. However, unlike in the voter model, the set of opinions is represented by the set of vertices of a finite connected graph that we call the opinion graph: when an individual interacts with a neighbor, she imitates this neighbor if and only if the distance between their opinions, defined as the graph distance induced by the opinion graph, does not exceed a certain confidence threshold. Our first result shows that, when the confidence threshold is at least equal to the radius of the opinion graph, the process fluctuates and clusters. We also establish a general sufficient condition for fixation of the process based on the structure of the opinion graph, which we then significantly improve for opinion graphs which are distance-regular. Our general results are used to understand the dynamics of the system for various examples of opinion graphs: paths and stars, which are not distance-regular, and cycles, hypercubes and the five Platonic solids, which are distance-regular.
\end{abstract}

\section{Introduction}

Since the work of Arratia (1983) on annihilating random walks, it is known that, for the one-dimensional voter model starting with infinitely many supporters of each opinion, there is fluctuation, i.e., the number of opinion changes at each vertex is almost surely infinite, as opposed to fixation, i.e., the number of opinion changes at each vertex is almost surely finite. The objective of this paper is to study the

Received by the editors September 9, 2015; accepted December 26, 2016.

2010 Mathematics Subject Classification. 60K35.

Key words and phrases. Interacting particle systems, voter model, opinion dynamics, confidence threshold, annihilating random walks, fluctuation, fixation, distance-regular graphs. 
dichotomy between fluctuation and fixation for a general class of opinion models with confidence threshold. The main novelty is to equip the set of opinions with the structure of a connected graph and use the induced graph distance to define mathematically a level of disagreement among individuals. Based on this modeling approach, some of the most popular models of opinion dynamics can be recovered by choosing the structure of the opinion space suitably: the constrained voter model, independently introduced in Itoh et al. (1998); Vazquez et al. (2003), is obtained by assuming that the opinion space is a path, while the Axelrod model for the dissemination of cultures (Axelrod, 1997) and the discrete Deffuant model (Deffuant et al., 2000) have also some connections with our model when the opinion space is a Hamming graph and a hypercube, respectively.

Model description - The class of models considered in this article are examples of interacting particle systems inspired from the voter model (Clifford and Sudbury, 1973; Holley and Liggett, 1975) for the dynamics of opinions. Individuals are located on the one-dimensional integer lattice and are characterized by their opinion, with the set of opinions being identified with the vertex set of a finite connected graph. The lattice represents the underlying spatial structure and is used to determine the interaction neighborhood of each individual. The finite connected graph, that we call the opinion graph, represents the structure of the opinion space and is used to determine the distance between two opinions and the level of disagreement among individuals. From now on, we call spatial distance the graph distance induced by the spatial structure and opinion distance the graph distance induced by the opinion graph. Individuals interact with each of their two nearest neighbors at rate one. As the result of an interaction, an individual imitates her neighbor if and only if the distance between their opinions just before the interaction does not exceed some confidence threshold $\tau \in \mathbb{N}$. More formally, we let

$$
\begin{aligned}
& \mathcal{G}:=(\mathbb{Z}, \mathcal{E})=\text { the spatial structure } \\
& \Gamma:=(V, E)=\text { the opinion graph }
\end{aligned}
$$

be respectively the one-dimensional lattice where each integer is connected to the previous and next integers by an edge and a general finite connected graph. Then, our opinion model is the continuous-time Markov chain whose state at time $t$ is a spatial configuration

$$
\eta_{t}: \mathbb{Z} \rightarrow V \text { where } \eta_{t}(x)=\text { opinion at } x \in \mathbb{Z} \text { at time } t
$$

and with transition rates at vertex $x \in \mathbb{Z}$ given for all $i, j \in V$ by

$$
\begin{aligned}
c_{i \rightarrow j}\left(x, \eta_{t}\right): & =\lim _{s \downarrow 0}(1 / s) P\left(\eta_{t+s}(x)=j \mid \eta_{t}=\eta \text { and } \eta(x)=i\right) \\
& =\operatorname{card}\left\{y \in N_{x}: \eta_{t}(y)=j\right\} \mathbf{1}\{d(i, j) \leq \tau\} .
\end{aligned}
$$

In addition, the transitions at different vertices of the spatial structure occur independently so there can be at most one opinion change at any given time. Here,

$$
N_{x}=\{y \in \mathbb{Z}:|x-y|=1\}=\{x-1, x+1\}
$$

denotes the interaction neighborhood of site $x$ while $d(i, j)$ refers to the opinion distance between opinion $i$ and opinion $j$, which is the length of the shortest path connecting both opinions on the opinion graph. Note that the classical voter model is simply obtained by assuming that the opinion graph consists of two vertices 
connected by an edge and that the confidence threshold equals one. The general class of opinion models (1.1) has been introduced in Scarlatos (2013).

Main results - The main question about the general opinion model is whether the system fluctuates and clusters, leading ultimately the population to a global consensus, or fixates in a fragmented configuration. Recall that the process

- fluctuates when $P\left(\eta_{t}(x)\right.$ changes infinitely often $)=1$ for all $x \in \mathbb{Z}$,

- fixates when $P\left(\eta_{t}(x)\right.$ changes a finite number of times $)=1$ for all $x \in \mathbb{Z}$,

- clusters when $\lim _{t \rightarrow \infty} P\left(\eta_{t}(x)=\eta_{t}(y)\right)=1$ for all $x, y \in \mathbb{Z}$.

Note that whether the system fluctuates and clusters or fixates in a fragmented configuration is sensitive to the initial configuration. Also, throughout the paper, we assume that the process starts from a product measure with densities which are constant across space, i.e.,

$$
\rho_{j}:=P\left(\eta_{0}(x)=j\right) \text { for all }(x, j) \in \mathbb{Z} \times V
$$

only depends on opinion $j$ but not on site $x$. To avoid trivialities, these densities are assumed to be positive. Sometimes, we will make the stronger assumption that all the opinions are equally likely at time zero. These two hypotheses correspond to the following two conditions:

$$
\begin{array}{lll}
\rho_{j}>0 & \text { for all } & j \in V \\
\rho_{j}=1 / F & \text { for all } & j \in V
\end{array}
$$

where $F:=$ card $V$ refers to the total number of opinions.

Key quantities to study the behavior of the system are the radius and the diameter of the opinion graph defined respectively as the minimum and maximum eccentricity of any vertex:

$$
\begin{aligned}
& \mathbf{r}:=\min _{i \in V} \max _{j \in V} d(i, j)=\text { the radius of the graph } \Gamma \\
& \mathbf{d}:=\max _{i \in V} \max _{j \in V} d(i, j)=\text { the diameter of the graph } \Gamma .
\end{aligned}
$$

Note that there is at least one pair $\left(i_{-}, i_{+}\right) \in V^{2}$ such that $d\left(i_{-}, i_{+}\right)=\mathbf{d}$. Later on, such vertices will be called antipodal. We also introduce the subset

$$
C(\Gamma, \tau):=\{i \in V: d(i, j) \leq \tau \text { for all } j \in V\}
$$

that we shall call the $\tau$-center of the opinion graph.

Using a coupling between the opinion model and a certain system of annihilating particles, we first prove the following sufficient condition for fluctuation and clustering of the process.

Theorem 1.1. Under assumption (1.2), we have the following two results.

a. The process fluctuates when

$$
d(i, j) \leq \tau \quad \text { for all } \quad(i, j) \in V_{1} \times V_{2}
$$

for some $V$-partition $\left\{V_{1}, V_{2}\right\}$.

b. The process clusters when the radius $\mathbf{r} \leq \tau$.

Except when the threshold is at least equal to the diameter, in which case the two conclusions of the theorem are trivial because the process reduces to a voter model with $F$ opinions, when the threshold is at least equal to the radius, both the $\tau$-center and its complement are nonempty, and therefore form a partition 
that satisfies (1.5) in the first part of the theorem. In particular, fluctuation also holds when the radius is not more than the threshold like in the second part of the theorem. Note however that the existence of a partition (1.5) does not imply that the radius is bounded from above by the threshold. For a counter-example, consider the cube in three dimensions. For this graph, there is a partition of the set of vertices that satisfies (1.5) with $\tau=2$ while the radius is equal to three. The other four Platonic solids also constitute counter-examples.

We now turn our attention to sufficient conditions for fixation of the system, beginning with general opinion graphs. At least for the process starting from the uniform product measure, these conditions can be expressed using

$$
N(\Gamma, s):=\operatorname{card}\{(i, j) \in V \times V: d(i, j)=s\} \quad \text { for } \quad s=1,2, \ldots, \mathbf{d},
$$

which is the number of pairs of opinions at opinion distance $s$ of each other. In the statement of the next theorem, the function $\lceil\cdot\rceil$ refers to the ceiling function.

Theorem 1.2. Fixation occurs in the following two cases.

a. Condition (1.3) holds and

$$
S(\Gamma, \tau):=\sum_{k>0}\left((k-2) \sum_{s:\lceil s / \tau\rceil=k} N(\Gamma, s)\right)>0 .
$$

b. There is a pair $\left(i_{-}, i_{+}\right)$of antipodal vertices and $\rho>0$ small such that

$$
\rho_{i_{-}}, \rho_{i_{+}}>1 / 2-\rho, \quad \rho_{i}<\rho \text { for all } i \neq i_{-}, i_{+} \quad \text { and } \mathbf{d}>2 \tau \text {. }
$$

Part $\mathrm{b}$ of the theorem implies that, when $\mathbf{d}>2 \tau$, there exist initial distributions that satisfy (1.2) from which the system fixates. This shows in particular that Theorems 1.1.a and 1.2.b give sharp conditions for graphs with $\mathbf{d}=2 \mathbf{r}$ in the following sense. For such opinion graphs,

- Theorem 1.1.a implies that, when $\mathbf{d} \leq 2 \tau$, the system starting from any initial distribution that satisfies (1.2) always fluctuates whereas

- Theorem 1.2.b implies that, when $\mathbf{d}>2 \tau$, there exist initial distributions that satisfy (1.2) from which the system fixates.

Examples of finite connected graphs such that $\mathbf{d}=2 \mathbf{r}$ are path graphs with an odd number of vertices and stars with branches of the same length.

Our last theorem, which is also the most challenging result of this paper, gives a significant improvement of the previous condition for fixation for distance-regular opinion graphs. This class of graphs is defined rigorously as follows: let

$$
\Gamma_{s}(i):=\{j \in V: d(i, j)=s\} \text { for } s=0,1, \ldots, \mathbf{d}
$$

be the distance partition of the vertex set $V$ for some $i \in V$. Then, the opinion graph is distance-regular if the so-called intersection numbers

$$
\begin{aligned}
& N\left(\Gamma,\left(i_{-}, s_{-}\right),\left(i_{+}, s_{+}\right)\right):=\operatorname{card}\left(\Gamma_{s_{-}}\left(i_{-}\right) \cap \Gamma_{s_{+}}\left(i_{+}\right)\right) \\
& =\operatorname{card}\left\{j \in V: d\left(i_{-}, j\right)=s_{-} \text {and } d\left(i_{+}, j\right)=s_{+}\right\} \\
& =f\left(s_{-}, s_{+}, d\left(i_{-}, i_{+}\right)\right)
\end{aligned}
$$

only depend on the distance $d\left(i_{-}, i_{+}\right)$but not on the particular choice of $i_{-}$and $i_{+}$. This implies that, for distance-regular opinion graphs, the number of vertices

$$
N(\Gamma,(i, s)):=\operatorname{card}\left(\Gamma_{s}(i)\right)=f(s, s, 0)=: h(s)
$$

does not depend on vertex $i$. To state our last theorem, we let

$$
\mathbf{W}(k):=-1+\sum_{1<n \leq k} \sum_{n \leq m \leq\lceil\mathbf{d} / \tau\rceil}\left(q_{n} q_{n+1} \cdots q_{m-1}\right) /\left(p_{n} p_{n+1} \cdots p_{m}\right)
$$


where we assume by convention an empty sum is equal to zero and an empty product is equal to one, and where the coefficients $p_{n}$ and $q_{n}$ are defined in terms of the intersection numbers as

$$
\begin{aligned}
& p_{n}:=\max \left\{\sum_{s:\lceil s / \tau\rceil=n-1} f\left(s_{-}, s_{+}, s\right) / h\left(s_{+}\right):\left\lceil s_{-} / \tau\right\rceil=1 \text { and }\left\lceil s_{+} / \tau\right\rceil=n\right\} \\
& q_{n}:=\min \left\{\sum_{s:\lceil s / \tau\rceil=n+1} f\left(s_{-}, s_{+}, s\right) / h\left(s_{+}\right):\left\lceil s_{-} / \tau\right\rceil=1 \text { and }\left\lceil s_{+} / \tau\right\rceil=n\right\} .
\end{aligned}
$$

Then, we have the following sufficient condition for fixation.

Theorem 1.3. Assume (1.3) and (1.7). Then, the process fixates when

$$
S_{\mathrm{reg}}(\Gamma, \tau):=\sum_{k>0}\left(\mathbf{W}(k) \sum_{s:\lceil s / \tau\rceil=k} h(s)\right)>0 .
$$

To understand the coefficients $p_{n}$ and $q_{n}$, note that, letting $i_{-}$and $j$ be two opinions at distance $s_{-}$of each other, we have the following interpretation:

$$
\begin{aligned}
f\left(s_{-}, s_{+}, s\right) / h\left(s_{+}\right)= & \text {probability that an opinion } i_{+} \text {chosen uniformly } \\
& \text { at random among the opinions at distance } s_{+} \text {from } \\
& \text { opinion } j \text { is at distance } s \text { from opinion } i_{-} .
\end{aligned}
$$

Outline of the proofs - The starting point of all our proofs is to use the formal machinery introduced in Lanchier (2012); Lanchier and Scarlatos (2013); Lanchier and Schweinsberg (2012) that consists in keeping track of the disagreements along the edges of the spatial structure. This idea has also been used in Lanchier and Moisson (2016); Lanchier and Scarlatos (2014) to study related models. In the context of our general opinion model, we put a pile of $s$ particles on edges that connect individuals who are at opinion distance $s$ of each other, i.e., we set

$$
\xi_{t}((x, x+1)):=d\left(\eta_{t}(x), \eta_{t}(x+1)\right) \text { for all } x \in \mathbb{Z} \text {. }
$$

The definition of the confidence threshold implies that piles with at most $\tau$ particles, that we call active, evolve according to symmetric random walks, while larger piles, that we call frozen, are static. In addition, the jump of an active pile onto another pile results in part of the particles being annihilated.

The main idea to prove fluctuation is to show that, after identifying opinions that belong to the same member of the partition (1.5), the process reduces to the voter model, and use that the one-dimensional voter model fluctuates according to Arratia (1983). Fluctuation, together with the stronger assumption $\mathbf{r} \leq \tau$, implies that the frozen piles, and ultimately all the piles of particles, go extinct, which is equivalent to clustering of the opinion model.

In contrast, fixation occurs when the frozen piles have a positive probability of never being reduced, which is more difficult to establish. To briefly explain our approach to prove fixation, we say that the pile at $(x, x+1)$ is of order $k$ when

$$
(k-1) \tau<\xi_{t}((x, x+1)) \leq k \tau .
$$

To begin with, we use a construction due to Bramson and Griffeath (1989) to obtain an implicit condition for fixation in terms of the initial number of piles of any given order in a large interval. Large deviation estimates for the number of such piles are then proved and used to turn this implicit condition into the explicit condition (1.6). To derive this condition, we use that at least $k-1$ active piles must jump onto a pile initially of order $k>1$ to turn this pile into an active pile. Condition (1.6) is obtained assuming the worst case scenario where the number of particles that annihilate is maximal.

To show the improved condition for fixation (1.8) for distance-regular opinion 
graphs, we use the same approach but count more carefully the number of annihilating events. First, we use duality-like techniques to prove that, when the opinion graph is distance-regular, the system of piles becomes Markov. This is used to prove that the jump of an active pile onto a pile of order $n>1$ reduces/increases its order with respective probabilities at most $p_{n}$ and at least $q_{n}$. This implies that the number of active piles that must jump onto a pile initially of order $k>1$ to turn it into an active pile is stochastically larger than the first hitting time to state 1 of a certain discrete-time birth and death process. This hitting time is equal in distribution to

$$
\sum_{1<n \leq k} \sum_{n \leq m \leq\lceil\mathbf{d} / \tau\rceil}\left(q_{n} q_{n+1} \cdots q_{m-1}\right) /\left(p_{n} p_{n+1} \cdots p_{m}\right)=1+\mathbf{W}(k) .
$$

The probabilities $p_{n}$ and $q_{n}$ are respectively the death parameter and the birth parameter of the discrete-time birth and death process while the integer $\lceil\mathbf{d} / \tau\rceil$ is the number of states of this process, which is also the maximum order of a pile.

\section{Application to specific opinion graphs}

In this section, we first apply our general results Theorems 1.1-1.3 to the opinion graphs which are represented in Figure 2.1 and then conclude with a conjecture for general opinion graphs inspired from the combination of our theoretical results and concrete examples.

To begin with, we look at paths and more generally stars with $b$ branches of equal length. For paths, one can think of the individuals as being characterized by their position about one issue, ranging from strongly agree to strongly disagree. For stars, individuals are offered $b$ alternatives: the center represents undecided individuals while vertices far from the center are more extremist in their position. These graphs are not distance-regular so we can only apply Theorem 1.2 to study fixation. This theorem combined with Theorem 1.1 gives the following two corollaries.

Corollary 2.1 (path). When $\Gamma$ is the path with $F$ vertices,

- the system fluctuates when (1.2) holds and $F \leq 2 \tau+1$ whereas

- the system fixates when (1.3) holds and

$$
3 F^{2}-(20 \tau+3) F+10(3 \tau+1) \tau>0 .
$$

Corollary 2.2 (star). When $\Gamma$ is the star with $b$ branches of length $r$,

- the system fluctuates when (1.2) holds and $r \leq \tau$ whereas

- the system fixates when (1.3) holds, $2 r>3 \tau$ and

$$
4(b-1) r^{2}+2((4-5 b) \tau+b-1) r+(6 b-5) \tau^{2}+(1-2 b) \tau>0 .
$$

To illustrate Theorem 1.3, we now look at distance-regular graphs, starting with the five convex regular polyhedra also known as the Platonic solids. These graphs are natural mathematically though we do not have any specific interpretation from the point of view of social sciences except, as explained below, for the cube and more generally hypercubes. For these five graphs, Theorems 1.1 and 1.3 give sharp results with the exact value of the critical threshold except for the dodecahedron for which the behavior when $\tau=3$ remains an open problem.

Corollary 2.3 (Platonic solids). Assume (1.3). Then, 


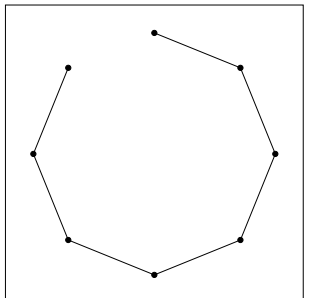

path with 8 vertices

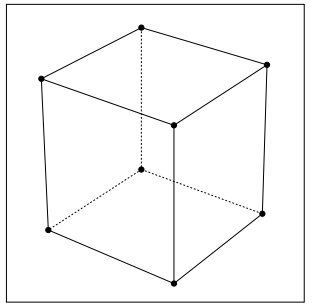

cube ( 6 faces, 8 vertices)

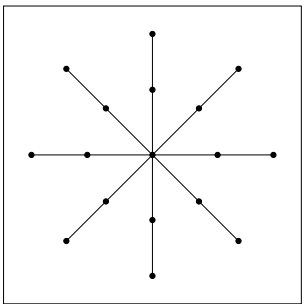

star with $b=8$ branches

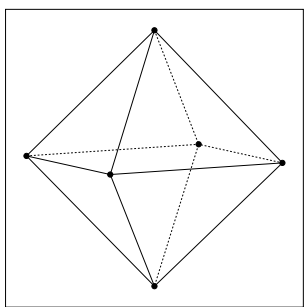

octahedron (8 faces, 6 vertices)

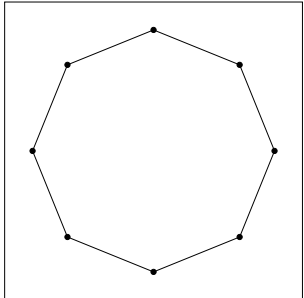

cycle with 8 vertices

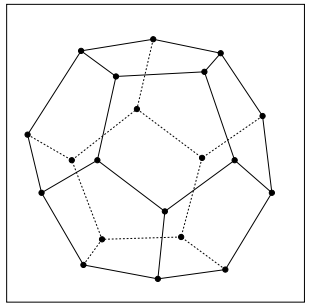

dodecahedron (12 faces, 20 vertices)

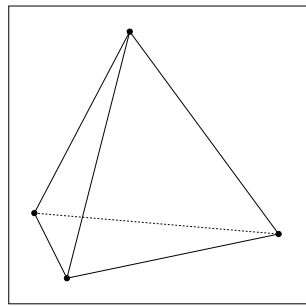

tetrahedron ( 4 faces, 4 vertices)

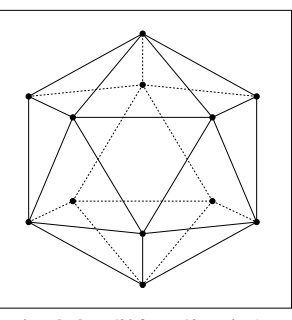

Figure 2.1. Opinion graphs considered in Corollaries 2.1-2.5

- the tetrahedral model fluctuates for all $\tau \geq 1$,

- the cubic model fluctuates when $\tau \geq 2$ and fixates when $\tau \leq 1$,

- the octahedral model fluctuates for all $\tau \geq 1$,

- the dodecahedral model fluctuates when $\tau \geq 4$ and fixates when $\tau \leq 2$,

- the icosahedral model fluctuates when $\tau \geq 2$ and fixates when $\tau \leq 1$.

Next, we look at the case where the individuals are characterized by some preferences represented by the set of vertices of a cycle. For instance, as explained in Boudourides and Scarlatos (2005), all strict orderings of three alternatives can be represented by the cycle with $3 !=6$ vertices.

Corollary 2.4 (cycle). When $\Gamma$ is the cycle with $F$ vertices,

- the system fluctuates when (1.2) holds and $F \leq 2 \tau+2$ whereas

- the system fixates when (1.3) hold and $F \geq 4 \tau+2$.

Finally, we look at hypercubes with $F=2^{d}$ vertices, which are generalizations of the three-dimensional cube. In this case, the individuals are characterized by their position - in favor or against - about $d$ different issues, and the opinion distance between two individuals is equal to the number of issues they disagree on. Theorem 1.3 gives the following result.

Corollary 2.5 (hypercube). When $\Gamma$ is the hypercube with $2^{d}$ vertices,

- the system fluctuates when (1.2) holds and $d \leq \tau+1$ whereas

- the system fixates when

$$
\text { (1.3) holds and } d / \tau>3 \text { or } d / \tau>2 \text { with } \tau \text { large. }
$$

Table 2.1 summarizes our results for the graphs of Figure 2.1. The second and third columns give the value of the radius and the diameter. The conditions in the fourth column are the conditions for fluctuation of the system obtained from the corollaries. For opinion graphs with a variable number of vertices, the last 


\begin{tabular}{|c|c|c|c|c|c|}
\hline opinion graph & radius & diameter & fluctuation & fix. $(\tau=1)$ & fix. ( $\tau$ large $)$ \\
\hline path & $\mathbf{r}=\lfloor F / 2\rfloor$ & $\mathbf{d}=F-1$ & $F \leq 2 \tau+1$ & $F \geq 6$ & $F / \tau>(10+\sqrt{10}) / 3 \approx 4.39$ \\
\hline $\operatorname{star}(b=3)$ & $\mathbf{r}=r$ & $\mathbf{d}=2 r$ & $r \leq \tau$ & $r \geq 2$ & $r / \tau>(11+\sqrt{17}) / 8 \approx 1.89$ \\
\hline $\operatorname{star}(b=5)$ & $\mathbf{r}=r$ & $\mathbf{d}=2 r$ & $r \leq \tau$ & $r \geq 2$ & $r / \tau>(21+\sqrt{41}) / 16 \approx 1.71$ \\
\hline cycle & $\mathbf{r}=\lfloor F / 2\rfloor$ & $\mathbf{d}=\lfloor F / 2\rfloor$ & $F \leq 2 \tau+2$ & $F \geq 6$ & $F / \tau>4$ \\
\hline hypercube & $\mathbf{r}=d$ & $\mathbf{d}=d$ & $d \leq \tau+1$ & $d \geq 3$ & $d / \tau>2$ \\
\hline opinion graph & radius & diameter & fluctuation & & fixation when \\
\hline tetrahedron & $\mathbf{r}=1$ & $\mathbf{d}=1$ & $\tau \geq 1$ & & $\tau=0$ \\
\hline cube & $\mathbf{r}=3$ & $\mathbf{d}=3$ & $\tau \geq 2$ & & $\tau \leq 1$ \\
\hline octahedron & $\mathbf{r}=2$ & $\mathbf{d}=2$ & $\tau \geq 1$ & & $\tau=0$ \\
\hline dodecahedron & $\mathbf{r}=5$ & $\mathbf{d}=5$ & $\tau \geq 4$ & & $\tau \leq 2$ \\
\hline icosahedron & $\mathbf{r}=3$ & $\mathbf{d}=3$ & $\tau \geq 2$ & & $\tau \leq 1$ \\
\hline
\end{tabular}

two columns give sufficient conditions for fixation in the two extreme cases when the confidence threshold is one and when the confidence threshold is large. To explain the last column for paths and stars, note that the opinion model fixates whenever $\mathbf{d} / \tau$ is larger than the largest root of the polynomials

$$
\begin{array}{ll}
3 X^{2}-20 X+30 & \text { for the path } \\
2 X^{2}-11 X+13 & \text { for the star with } b=3 \text { branches } \\
4 X^{2}-21 X+25 & \text { for the star with } b=5 \text { branches }
\end{array}
$$

and the diameter of the opinion graph is sufficiently large. These polynomials are obtained from the conditions in Corollaries $2.1-2.2$ by only keeping the terms with degree two.

Conjectures about whether the process fluctuates or fixates are difficult to obtain from numerical simulations because the process always fixates when the spatial structure is finite. The distinction between fluctuation and fixation can also be determined by looking at the average domain length in the final configuration but here again realizations of the finite system are difficult to interpret and may not be symptomatic of the behavior of the infinite system. The approaches which aid towards the estimation of the critical threshold constitute challenging computational problems. However, based on our results, we conjecture that, provided condition (1.2) holds, the process

$$
\text { fluctuates when } \mathbf{d} \leq 2 \tau \text { and fixates when } \mathbf{d}>2 \tau \text {. }
$$

Note that this conjecture is supported by the discussion right after Theorem 1.2 which shows that, for opinion graphs with $\mathbf{d}=2 \mathbf{r}$, the process fluctuates starting from all possible initial distributions that satisfy (1.2) if and only if $\mathbf{d} \leq 2 \tau$. Note also that (2.1) is consistent with all the results summarized in Table 2.1, though, for technical reasons, the fixation results are only proved for the process starting from a uniform product measure. In particular, the conjecture gives indeed the sharp results obtained for the tetrahedron, cube, octahedron and icosahedron, which are examples of graphs with $\mathbf{d}=\mathbf{r}$. 


\section{Coupling with a system of annihilating particles}

To study the general opinion model, it is convenient to construct the process from a graphical representation and to introduce a coupling between the opinion model and a certain system of annihilating particles that keeps track of the discrepancies along the edges of the lattice rather than the opinion at each vertex. This system of particles can also be constructed from the same graphical representation which consists of the following collection of independent Poisson processes:

- For each $x \in \mathbb{Z}$, let $\left(N_{t}(x, x \pm 1): t \geq 0\right)$ be a rate one Poisson process.

- Let $T_{n}(x, x \pm 1):=\inf \left\{t: N_{t}(x, x \pm 1)=n\right\}$ be its $n$th arrival time.

This collection of independent Poisson processes is then turned into a percolation structure by drawing an arrow $x \rightarrow x \pm 1$ at time $t:=T_{n}(x, x \pm 1)$. We say that this arrow is active when

$$
d\left(\eta_{t-}(x), \eta_{t-}(x \pm 1)\right) \leq \tau .
$$

The configuration at time $t$ is then obtained by setting

$$
\begin{aligned}
\eta_{t}(x \pm 1) & =\eta_{t-}(x) & & \text { when the arrow } x \rightarrow x \pm 1 \text { is active } \\
& =\eta_{t-}(x \pm 1) & & \text { when the arrow } x \rightarrow x \pm 1 \text { is not active }
\end{aligned}
$$

and leaving the opinion at all the other vertices unchanged. An argument due to Harris Harris (1972) implies that the opinion model starting from any configuration can indeed be constructed using this percolation structure and rule (3.1). From the collection of active arrows, we construct active paths as in percolation theory. More precisely, we say that there is an active path from $(z, s)$ to $(x, t)$, and write $(z, s) \leadsto(x, t)$, whenever there exist

$$
s_{0}=s<s_{1}<\cdots<s_{n+1}=t \quad \text { and } \quad x_{0}=z, x_{1}, \ldots, x_{n}=x
$$

such that the following two conditions hold:

- For $j=1,2, \ldots, n$, there is an active arrow $x_{j-1} \rightarrow x_{j}$ at time $s_{j}$.

- For $j=0,1, \ldots, n$, there is no active arrow pointing at $\left\{x_{j}\right\} \times\left(s_{j}, s_{j+1}\right)$.

These two conditions imply that

for all $(x, t) \in \mathbb{Z} \times \mathbb{R}_{+}$there is a unique $z \in \mathbb{Z}$ such that $(z, 0) \leadsto(x, t)$.

Moreover, because of the definition of active arrows, the opinion at vertex $x$ at time $t$ originates from and is therefore equal to the initial opinion at vertex $z$ so we call vertex $z$ the ancestor of vertex $x$ at time $t$.

As previously mentioned, to study the general opinion model, we look at the process that keeps track of the discrepancies along the edges rather than the actual opinion at each vertex, that we shall call the system of piles. To define this process, it is convenient to identify each edge with its midpoint and to define translations on the edge set as follows:

$$
\begin{aligned}
& e:=\{x, x+1\} \equiv x+1 / 2 \quad \text { for all } \quad x \in \mathbb{Z} \\
& e+v:=\{x, x+1\}+v \equiv x+1 / 2+v \quad \text { for all } \quad(x, v) \in \mathbb{Z} \times \mathbb{R} \text {. }
\end{aligned}
$$

The system of piles is then defined as

$$
\xi_{t}(e):=d\left(\eta_{t}(e-1 / 2), \eta_{t}(e+1 / 2)\right) \quad \text { for all } \quad e \in \mathbb{Z}+1 / 2,
$$


and it is convenient to think of edge $e$ as being occupied by a pile of $\xi_{t}(e)$ particles. The dynamics of the opinion model induces the following evolution rules on this system of particles. Assuming that there is an arrow $x-1 \rightarrow x$ at time $t$ and that

$$
\begin{aligned}
& \xi_{t-}(x-1 / 2):=d\left(\eta_{t-}(x), \eta_{t-}(x-1)\right)=s_{-} \\
& \xi_{t-}(x+1 / 2):=d\left(\eta_{t-}(x), \eta_{t-}(x+1)\right)=s_{+}
\end{aligned}
$$

we have the following alternative:

- In case $s_{-}=0$ (there is no particle on the edge), the two interacting agents already agree just before the interaction therefore nothing happens.

- In case $s_{-}>\tau$ (there are more than $\tau$ particles on the edge), the two agents disagree too much to trust each other so nothing happens.

- In case $0<s_{-} \leq \tau$ (there is at least one but no more than $\tau$ particles on the edge), the agent at vertex $x$ mimics her left neighbor, which gives

$$
\begin{aligned}
\xi_{t}(x-1 / 2) & :=d\left(\eta_{t}(x), \eta_{t}(x-1)\right) \\
& =d\left(\eta_{t-}(x-1), \eta_{t-}(x-1)\right)=0 \\
\xi_{t}(x+1 / 2) & :=d\left(\eta_{t}(x), \eta_{t}(x+1)\right) \\
& =d\left(\eta_{t-}(x-1), \eta_{t-}(x+1)\right) .
\end{aligned}
$$

In particular, there is no more particles at edge $x-1 / 2$. In addition, the size $s$ of the pile at edge $x+1 / 2$ at time $t$, where size of a pile refers to the number of particles in that pile, satisfies the two inequalities

$$
\begin{aligned}
s & \leq\left|d\left(\eta_{t-}(x-1), \eta_{t-}(x)\right)+d\left(\eta_{t-}(x), \eta_{t-}(x+1)\right)\right| \\
& =\left|s_{-}+s_{+}\right| \\
s & \geq\left|d\left(\eta_{t-}(x-1), \eta_{t-}(x)\right)-d\left(\eta_{t-}(x), \eta_{t-}(x+1)\right)\right| \\
& =\left|s_{-}-s_{+}\right| .
\end{aligned}
$$

Note that the first inequality implies that the process involves deaths of particles but no births, which is a key property that will be used later.

Similar evolution rules are obtained by exchanging the direction of the interaction from which we deduce the following description for the dynamics of piles:

- Piles with more than $\tau$ particles cannot move: we call such piles frozen piles and the particles in such piles frozen particles.

- Piles with at most $\tau$ particles jump one unit to the left or to the right at rate one: we call such piles active piles and the particles in such piles active particles. Note that arrows in the graphical representation are active if and only if they cross an active pile.

- When a pile of size $s_{-}$jumps onto a pile of size $s_{+}$this results in a pile whose size $s$ satisfies the two inequalities in (3.2) so we say that $s_{-}+s_{+}-s$ particles are annihilated.

\section{Proof of Theorem 1.1}

Before proving the theorem, we start with some preliminary remarks. To begin with, we observe that, when the diameter $\mathbf{d} \leq \tau$, the $\tau$-center covers all the opinion graph, indicating that the model reduces to a multitype voter model with $F=$ 
card $V$ opinions, in which case the theorem is trivial. To prove the theorem in the nontrivial case $\tau<\mathbf{d}$, we introduce the set

$$
B(\Gamma, \tau):=\{i \in V: d(i, j)>\tau \text { for some } j \in V\}
$$

that we call the $\tau$-boundary of the opinion graph. One key ingredient to our proof is the following lemma, which gives a sufficient condition for (1.5) to hold.

Lemma 4.1. $V_{1}=C(\Gamma, \tau)$ and $V_{2}=B(\Gamma, \tau)$ satisfy (1.5) when $\mathbf{r} \leq \tau<\mathbf{d}$.

Proof: From (1.4) and (4.1), we get $B(\Gamma, \tau)=V \backslash C(\Gamma, \tau)$ therefore

$$
C(\Gamma, \tau) \cup B(\Gamma, \tau)=V \quad \text { and } \quad C(\Gamma, \tau) \cap B(\Gamma, \tau)=\varnothing .
$$

In addition, the $\tau$-center of the graph is nonempty because

$$
\begin{aligned}
& C(\Gamma, \tau) \neq \varnothing \quad \text { if and only if there is } i \in V \text { such that } \\
& \qquad d(i, j) \leq \tau \text { for all } j \in V \\
& \text { if and only if there is } i \in V \text { such that } \\
& \qquad \max _{j \in V} d(i, j) \leq \tau \\
& \text { if and only if } \min _{i \in V} \max _{j \in V} d(i, j) \leq \tau \\
& \text { if and only if } \mathbf{r} \leq \tau
\end{aligned}
$$

while the $\tau$-boundary is nonempty because

$$
\begin{aligned}
B(\Gamma, \tau) \neq \varnothing \quad & \text { if and only if there is } i \in V \text { such that } \\
\qquad d(i, j)>\tau \text { for some } j \in V & \\
& \text { if and only if there are } i, j \in V \text { such that } d(i, j)>\tau \\
& \text { if and only if } \max _{i \in V} \max _{j \in V} d(i, j)>\tau \\
& \text { if and only if } \mathbf{d}>\tau .
\end{aligned}
$$

This shows that $\left\{V_{1}, V_{2}\right\}$ is a partition of the set of opinions. Finally, since all the vertices in the $\tau$-center are within distance $\tau$ of all the other vertices, we also have that condition (1.5) holds.

The previous lemma will be used in the proof of part b. From now on, we call vertices in the $\tau$-center the centrist opinions and vertices in the $\tau$-boundary the extremist opinions.

Proof of Theorem 1.1.a (fluctuation). Under condition (1.5), agents who support an opinion in the set $V_{1}$ are within the confidence threshold of agents who support an opinion in $V_{2}$, therefore the transition rates (1.1) imply that

$$
\begin{aligned}
c_{i \rightarrow j}\left(x, \eta_{t}\right) & =\lim _{s \downarrow 0}(1 / s) P\left(\eta_{t+s}(x)=j \mid \eta_{t} \text { and } \eta_{t}(x)=i\right) \\
& =\operatorname{card}\left\{y \in N_{x}: \eta_{t}(y)=j\right\}
\end{aligned}
$$

for every $(i, j) \in V_{1} \times V_{2}$ and every $(i, j) \in V_{2} \times V_{1}$. Let

$$
\zeta_{t}(x):=\mathbf{1}\left\{\eta_{t}(x) \in V_{2}\right\} \quad \text { for all } \quad x \in \mathbb{Z} .
$$

Since, according to (4.3), we have

- for all $j \in V_{2}$, the rates $c_{i \rightarrow j}\left(x, \eta_{t}\right)$ are constant across all $i \in V_{1}$,

- for all $i \in V_{1}$, the rates $c_{j \rightarrow i}\left(x, \eta_{t}\right)$ are constant across all $j \in V_{2}$, 
the process $\left(\zeta_{t}\right)$ is Markov with transition rates

$$
\begin{aligned}
c_{0 \rightarrow 1}\left(x, \zeta_{t}\right) & :=\lim _{s \downarrow 0}(1 / s) P\left(\zeta_{t+s}(x)=1 \mid \zeta_{t} \text { and } \zeta_{t}(x)=0\right) \\
& =\sum_{i \in V_{1}} \sum_{j \in V_{2}} c_{i \rightarrow j}\left(x, \eta_{t}\right) P\left(\eta_{t}(x)=i \mid \zeta_{t}(x)=0\right) \\
& =\sum_{i \in V_{1}} \sum_{j \in V_{2}} \operatorname{card}\left\{y \in N_{x}: \eta_{t}(y)=j\right\} P\left(\eta_{t}(x)=i \mid \zeta_{t}(x)=0\right) \\
& =\sum_{j \in V_{2}} \operatorname{card}\left\{y \in N_{x}: \eta_{t}(y)=j\right\}=\operatorname{card}\left\{y \in N_{x}: \zeta_{t}(y)=1\right\}
\end{aligned}
$$

and similarly for the reverse transition

$$
c_{1 \rightarrow 0}\left(x, \zeta_{t}\right)=\operatorname{card}\left\{y \in N_{x}: \eta_{t}(y) \in V_{1}\right\}=\operatorname{card}\left\{y \in N_{x}: \zeta_{t}(y)=0\right\} .
$$

This shows that $\left(\zeta_{t}\right)$ is the voter model. In addition, since $V_{1}, V_{2} \neq \varnothing$,

$$
P\left(\zeta_{0}(x)=0\right)=P\left(\eta_{0}(x) \in V_{1}\right)=\sum_{j \in V_{1}} \rho_{j} \in(0,1)
$$

whenever condition (1.2) holds. In particular, the result follows from the fact that the one-dimensional voter model starting with a positive density of each type fluctuates. This last result is a consequence of site recurrence for annihilating random walks proved in Arratia (1983).

Proof of Theorem 1.1.b (clustering). Since $\mathbf{r} \leq \tau<\mathbf{d}$,

$$
V_{1}=C(\Gamma, \tau) \text { and } \quad V_{2}=B(\Gamma, \tau)
$$

form a partition of $V$ according to Lemma 4.1. This implies that, not only the opinion model fluctuates, but also the coupled voter model (4.4) for this specific partition fluctuates, which is the key to the proof. First, we define the function

$$
u(t):=E\left(\xi_{t}(e)\right)=\sum_{0 \leq j \leq \mathbf{d}} j P\left(\xi_{t}(e)=j\right)
$$

which, in view of translation invariance of the initial configuration and the evolution rules, does not depend on the choice of $e$. Note that, since the system of particles coupled with the process involves deaths of particles but no births, the function $u(t)$ is nonincreasing in time. Since it is also nonnegative, it has a limit: $u(t) \rightarrow l$ as $t \rightarrow \infty$. Now, on the event that an edge $e$ is occupied by a pile of at least one particle at a given time $t$, we have the following alternative:

(1) In case edge $e:=x+1 / 2$ carries a frozen pile, since the centrist agents are within the confidence threshold of all the other individuals, we have

$$
\eta_{t}(x) \in V_{2}=B(\Gamma, \tau) \quad \text { and } \quad \eta_{t}(x+1) \in V_{2}=B(\Gamma, \tau) .
$$

Now, using that the voter model (4.4) fluctuates,

$$
\begin{aligned}
T:=\inf \{s>t: & \eta_{s}(x) \in V_{1}=C(\Gamma, \tau) \\
& \text { or } \left.\eta_{s}(x+1) \in V_{1}=C(\Gamma, \tau)\right\}<\infty
\end{aligned}
$$

almost surely, while by definition of the $\tau$-center, we have

$$
\xi_{T}(e)=d\left(\eta_{T}(x), \eta_{T}(x+1)\right) \leq \tau<\xi_{t}(e) .
$$

In particular, with probability one, at least one of the frozen particles at $e$ is annihilated eventually.

(2) In case edge $e:=x+1 / 2$ carries an active pile, since one-dimensional symmetric random walks are recurrent, this pile eventually intersects another pile. Let $s_{-}$and $s_{+}$be respectively the size of these two piles and let $s$ be the size of the pile of particles resulting from their intersection. Then, we have the following alternative: 
(a) In case $s<s_{-}+s_{+}$and $s>\tau$, at least one particle is annihilated and there is either formation or increase of a frozen pile so we are back to case (1): since the voter model coupled with the opinion model fluctuates, with probability one, at least one of the frozen particles in this pile is annihilated eventually.

(b) In case $s<s_{-}+s_{+}$and $s \leq \tau$, with probability one, at least one particle is annihilated.

(c) In case $s=s_{-}+s_{+}$and $s>\tau$, there is either formation or increase of a frozen pile so we are back to case (1): since the voter model coupled with the opinion model fluctuates, with probability one, at least one of the frozen particles in this pile is annihilated eventually.

(d) In case $s=s_{-}+s_{+}$and $s \leq \tau$, the resulting pile is again active so it keeps moving until, after a finite number of collisions, we are back to either (a) or (b) or (c) and, with probability one, at least one particle is annihilated eventually.

This shows that, as long as $u(t)$ is positive, it is also decreasing. In particular, there exists an increasing sequence of deterministic times $\left(t_{n}\right)_{n \in \mathbb{N}} \subset \mathbb{R}_{+}$such that

$$
u\left(t_{n}\right) \leq(1 / 2) u\left(t_{n-1}\right) \leq(1 / 4) u\left(t_{n-2}\right) \leq \cdots \leq(1 / 2)^{n} u(0) \leq(1 / 2)^{n} F
$$

from which it follows that the density of particles decreases to zero:

$$
\lim _{t \rightarrow \infty} P\left(\xi_{t}(e) \neq 0\right) \leq \lim _{t \rightarrow \infty} u(t)=0 \text { for all } e \in \mathbb{Z}+1 / 2 .
$$

In conclusion, for all $x, y \in \mathbb{Z}$ with $x<y$, we have

$$
\begin{aligned}
\lim _{t \rightarrow \infty} P\left(\eta_{t}(x) \neq \eta_{t}(y)\right) & \leq \lim _{t \rightarrow \infty} P\left(\xi_{t}(z+1 / 2) \neq 0 \text { for some } x \leq z<y\right) \\
& \leq \lim _{t \rightarrow \infty} \sum_{x \leq z<y} P\left(\xi_{t}(z+1 / 2) \neq 0\right) \\
& =(y-x) \lim _{t \rightarrow \infty} P\left(\xi_{t}(e) \neq 0\right)=0,
\end{aligned}
$$

which proves clustering.

\section{Sufficient condition for fixation}

This section and the next two ones are devoted to the proof of Theorem 1.2 which focuses on the fixation regime. In this section, we give a general sufficient condition for fixation that can be expressed based on the initial number of active particles and frozen particles in a large random interval. The main ingredient of the proof is a construction due to Bramson and Griffeath (1989) based on duality-like techniques looking at active paths. The next section establishes large deviation estimates for the initial number of particles in order to simplify the condition for fixation using instead the expected number of active and frozen particles per edge. This is used in the subsequent section to prove Theorem 1.2. The next lemma gives a condition for fixation based on properties of the active paths, which is the analog of Bramson and Griffeath (1989, Lemma 2).

Lemma 5.1. For all $z \in \mathbb{Z}$, let

$$
T(z):=\inf \{t:(z, 0) \leadsto(0, t)\} .
$$

Then, the opinion model fixates whenever

$$
\lim _{N \rightarrow \infty} P(T(z)<\infty \text { for some } z<-N)=0 .
$$


Proof: This follows the proof of Lanchier and Scarlatos (2013, Lemma 4).

To derive a more explicit condition for fixation, we let

$$
H_{N}:=\{T(z)<\infty \text { for some } z<-N\}
$$

be the event introduced in (5.1). Following the construction in Bramson and Griffeath (1989), we also let $\tau_{N}$ be the first time an active path starting from the left of $-N$ hits the origin, and observe that

$$
\tau_{N}=\inf \{T(z): z \in(-\infty,-N)\} .
$$

In particular, the event $H_{N}$ can be written as

$$
H_{N}=\bigcap_{z<-N}\{T(z)<\infty\}=\left\{\tau_{N}<\infty\right\} .
$$

Given the event $H_{N}$, we let $z_{-}<-N$ and $z_{+} \geq 0$ be the initial position of the active path and the rightmost source of an active path that reaches the origin by time $\tau_{N}$. More formally, we define

$$
\begin{aligned}
& z_{-}:=\min \left\{z \in \mathbb{Z}:(z, 0) \leadsto\left(0, \tau_{N}\right)\right\}<-N \\
& z_{+}:=\max \left\{z \in \mathbb{Z}:(z, 0) \leadsto\left(0, \sigma_{N}\right) \text { for some } \sigma_{N}<\tau_{N}\right\} \geq 0,
\end{aligned}
$$

and let $I_{N}=\left(z_{-}, z_{+}\right)$. Now, we observe that, on the event $H_{N}$,

- All the frozen piles initially in $I_{N}$ have been destroyed, i.e., turned into active piles due to the occurrence of annihilating events, by time $\tau_{N}$.

- The active particles initially outside the interval $I_{N}$ cannot jump inside the space-time region delimited by the two active paths implicitly defined in (5.3) because the existence of such particles would contradict the minimality of $z_{-}$or the maximality of $z_{+}$.

This, together with equation (5.2), implies that, given the event $H_{N}$, all the frozen piles initially in the random interval $I_{N}$ must have been destroyed by either active piles initially in this interval or active piles that result from the destruction of these frozen piles. To quantify this statement, we attach random variables, that we call contributions, to each edge. The definition depends on whether the edge initially carries an active pile or a frozen pile. To begin with, we give an arbitrary deterministic contribution, say -1 , to each pile initially active by setting

$$
\operatorname{cont}(e):=-1 \quad \text { whenever } \quad 0<\xi_{0}(e) \leq \tau \text {. }
$$

Now, we observe that, given $H_{N}$, for each frozen pile initially in $I_{N}$, a random number of active piles must have jumped onto this frozen pile to turn it into an active pile. Therefore, to define the contribution of a frozen pile, we let

$$
T_{e}:=\inf \left\{t>0: \xi_{t}(e) \leq \tau\right\}
$$

and define the contribution of a frozen pile initially at $e$ as

$$
\operatorname{cont}(e):=-1+\text { number of active piles that hit } e \text { until time } T_{e} \text {. }
$$

Note that (5.6) reduces to (5.4) when edge $e$ carries initially an active pile since in this case the time until the edge becomes active is zero, therefore (5.6) can be used as the general definition for the contribution of an edge with at least one particle. Edges with initially no particle have contribution zero. Since the occurrence of $H_{N}$ implies that all the frozen piles initially in $I_{N}$ must have been destroyed by either active piles initially in this interval or active piles that result from the destruction 
of these frozen piles, in which case $T_{e}<\infty$ for all the edges in the interval, and since particles in an active pile jump all at once rather than individually,

$$
\begin{aligned}
H_{N} & \subset\left\{\sum_{e \in I_{N}} \operatorname{cont}\left(e \mid T_{e}<\infty\right) \leq 0\right\} \\
& \subset\left\{\sum_{e \in(l, r)} \operatorname{cont}\left(e \mid T_{e}<\infty\right) \leq 0 \text { for some } l<-N \text { and some } r \geq 0\right\} .
\end{aligned}
$$

Lemma 5.1 and (5.7) are used in section 7 together with the large deviation estimates for the number of active and frozen piles showed in the following section to prove Theorem 1.2.

\section{Large deviation estimates}

The objective of this section is to give large deviation estimates for the total contribution of a large interval, which will be used later to find a good upper bound for the probability in (5.1) and deduce a sufficient condition for fixation of the opinion model. In order to state our result, we say that an edge is of type $i \rightarrow j$ when it connects an individual with initial opinion $i$ on the left to an individual with initial opinion $j$ on the right, and let

$$
e_{N}(i \rightarrow j):=\operatorname{card}\left\{x \in(0, N): \eta_{0}(x)=i \text { and } \eta_{0}(x+1)=j\right\}
$$

denote the number of edges of type $i \rightarrow j$ in the interval $(0, N)$. Since large deviation holds not only for the contribution defined in the previous section but also for any function of the edge type, we state our result in this more general context.

Lemma 6.1. Let $w: V \times V \rightarrow \mathbb{R}$ be any function such that

$$
w(i, i)=0 \quad \text { for all } i \in V
$$

and let $W: \mathbb{Z}+1 / 2 \rightarrow \mathbb{R}$ be the function defined as

$$
W_{e}:=w(i, j) \quad \text { whenever edge } e \text { is of type } i \rightarrow j .
$$

Then, for all $\epsilon>0$, there exists $c_{0}>0$ such that

$$
P\left(\sum_{e \in(0, N)}\left(W_{e}-E\left(W_{e}\right)\right) \notin(-\epsilon N, \epsilon N)\right) \leq \exp \left(-c_{0} N\right) \quad \text { for all } N \text { large. }
$$

Proof: Even though the types of consecutive edges are not independent, since the initial opinions are independent, for all $\epsilon^{\prime}>0$, there exists $c_{1}>0$ such that

$$
\begin{aligned}
& P\left(e_{N}(i \rightarrow j)-N \rho_{i} \rho_{j} \notin\left(-\epsilon^{\prime} N, \epsilon^{\prime} N\right)\right) \\
& \quad=P\left(e_{N}(i \rightarrow j)-E\left(e_{N}(i \rightarrow j)\right) \notin\left(-\epsilon^{\prime} N, \epsilon^{\prime} N\right)\right) \leq \exp \left(-c_{1} N\right)
\end{aligned}
$$

for all $N$ large and all $i \neq j$. In other respects,

$$
\begin{aligned}
& \sum_{e \in(0, N)}\left(W_{e}-E\left(W_{e}\right)\right)=\sum_{e \in(0, N)} W_{e}-N E\left(W_{e}\right) \\
& \quad=\sum_{i \neq j} w(i, j) e_{N}(i \rightarrow j)-N \sum_{i \neq j} w(i, j) P(e \text { is of type } i \rightarrow j) \\
& \quad=\sum_{i \neq j} w(i, j)\left(e_{N}(i \rightarrow j)-N \rho_{i} \rho_{j}\right) .
\end{aligned}
$$

Finally, letting

$$
m:=\max _{i \neq j}|w(i, j)|<\infty \quad \text { and } \quad \epsilon^{\prime}:=1 / m F^{2},
$$


it follows from (6.1)-(6.2) that

$$
\begin{aligned}
& P\left(\sum_{e \in(0, N)}\left(W_{e}-E\left(W_{e}\right)\right) \notin(-\epsilon N, \epsilon N)\right) \\
& \quad=P\left(\sum_{i \neq j} w(i, j)\left(e_{N}(i \rightarrow j)-N \rho_{i} \rho_{j}\right) \notin(-\epsilon N, \epsilon N)\right) \\
& \quad \leq P\left(w(i, j)\left(e_{N}(i \rightarrow j)-N \rho_{i} \rho_{j}\right) \notin\left(-\epsilon N / F^{2}, \epsilon N / F^{2}\right) \text { for some } i \neq j\right) \\
& \quad \leq P\left(e_{N}(i \rightarrow j)-N \rho_{i} \rho_{j} \notin\left(-\epsilon N / m F^{2}, \epsilon N / m F^{2}\right) \text { for some } i \neq j\right) \\
& \quad=P\left(e_{N}(i \rightarrow j)-N \rho_{i} \rho_{j} \notin\left(-\epsilon^{\prime} N, \epsilon^{\prime} N\right) \text { for some } i \neq j\right) \leq F^{2} \exp \left(-c_{1} N\right)
\end{aligned}
$$

for all $N$ large. This completes the proof.

\section{Proof of Theorem 1.2 (general opinion graphs)}

The key ingredients to prove Theorem 1.2 are Lemma 5.1 and inclusions (5.7). The large deviation estimates of the previous section are also important to make the sufficient condition for fixation more explicit and applicable to particular opinion graphs. First, we find a lower bound $W_{e}$, that we shall call weight, for the contribution of any given edge $e$. This lower bound is deterministic given the initial number of particles at the edge and is obtained assuming the worst case scenario where all the active piles annihilate with frozen piles rather than other active piles. More precisely, we have the following lemma.

Lemma 7.1. For all $k>0$,

$$
\operatorname{cont}\left(e \mid T_{e}<\infty\right) \geq W_{e}:=k-2 \text { when }(k-1) \tau<\xi_{0}(e) \leq k \tau \text {. }
$$

Proof: The jump of an active pile of size $s_{-} \leq \tau$ onto a frozen pile of size $s_{+}>\tau$ decreases the size of this frozen pile by at most $s_{-}$particles. Since in addition active piles have at most $\tau$ particles, whenever the initial number of frozen particles at edge $e$ satisfies

$$
(k-1) \tau<\xi_{0}(e) \leq k \tau \quad \text { for some } \quad k \geq 2,
$$

at least $k-1$ active piles must have jumped onto $e$ until time $T_{e}<\infty$. Recalling (5.6) gives the result when edge $e$ carries a frozen while the result is trivial when the edge carries an active pile since, in this case, both its contribution and its weight are equal to -1 .

In view of Lemma 7.1, it is convenient to classify piles depending on the number of complete blocks of $\tau$ particles they contain: we say that the pile at $e$ is of order $k>0$ when

$$
(k-1) \tau<\xi_{t}(e) \leq k \tau \quad \text { or equivalently } \quad\left\lceil\xi_{t}(e) / \tau\right\rceil=k
$$

so that active piles are exactly the piles of order one and the weight of a pile is simply its order minus two. Now, we note that Lemma 7.1 and (5.7) imply that

$$
H_{N} \subset\left\{\sum_{e \in(l, r)} W_{e} \leq 0 \text { for some } l<-N \text { and some } r \geq 0\right\} .
$$

Since in addition fixation occurs when $\lim _{N \rightarrow \infty} P\left(H_{N}\right)=0$ according to Lemma 5.1, the main objective to prove fixation is to find an upper bound that converges to zero as $N \rightarrow \infty$ for the probability of the event on the right-hand side of (7.2). This is the key to proving the following general fixation result from which both parts of Theorem 1.2 can be easily deduced. 
Lemma 7.2. Assume (1.2). Then, the system fixates whenever

$$
\sum_{i, j \in V} \rho_{i} \rho_{j} \sum_{k>0}\left((k-2) \sum_{s:\lceil s / \tau\rceil=k} \mathbf{1}\{d(i, j)=s\}\right)>0 .
$$

Proof: To begin with, we observe that

$$
\begin{aligned}
P\left(\xi_{0}(e)=s\right) & =\sum_{i, j \in V} P\left(\eta_{0}(x)=i \text { and } \eta_{0}(x+1)=j\right) \mathbf{1}\{d(i, j)=s\} \\
& =\sum_{i, j \in V} \rho_{i} \rho_{j} \mathbf{1}\{d(i, j)=s\} .
\end{aligned}
$$

Recalling (7.1), it follows that

$$
\begin{aligned}
E\left(W_{e}\right) & =\sum_{k>0}(k-2) P\left((k-1) \tau<\xi_{0}(e) \leq k \tau\right) \\
& =\sum_{k>0}\left((k-2) \sum_{(k-1) \tau<s \leq k \tau} P\left(\xi_{0}(e)=s\right)\right) \\
& =\sum_{k>0}\left((k-2) \sum_{(k-1) \tau<s \leq k \tau} \sum_{i, j \in V} \rho_{i} \rho_{j} \mathbf{1}\{d(i, j)=s\}\right) \\
& =\sum_{i, j \in V} \rho_{i} \rho_{j} \sum_{k>0}\left((k-2) \sum_{s:\lceil s / \tau\rceil=k} \mathbf{1}\{d(i, j)=s\}\right)
\end{aligned}
$$

which is positive under the assumption of the lemma. In particular, applying the large deviation estimate in Lemma 6.1 with $\epsilon:=E\left(W_{e}\right)>0$, we deduce that

$$
\begin{aligned}
& P\left(\sum_{e \in(0, N)} W_{e} \leq 0\right)=P\left(\sum_{e \in(0, N)}\left(W_{e}-E\left(W_{e}\right)\right) \leq-\epsilon N\right) \\
& \leq P\left(\sum_{e \in(0, N)}\left(W_{e}-E\left(W_{e}\right)\right) \notin(-\epsilon N, \epsilon N)\right) \leq \exp \left(-c_{0} N\right)
\end{aligned}
$$

for all $N$ large, which, in turn, implies with (7.2) that

$$
\begin{aligned}
P\left(H_{N}\right) & \leq P\left(\sum_{e \in(l, r)} W_{e} \leq 0 \text { for some } l<-N \text { and } r \geq 0\right) \\
& \leq \sum_{l<-N} \sum_{r \geq 0} \exp \left(-c_{0}(r-l)\right) \rightarrow 0
\end{aligned}
$$

as $N \rightarrow \infty$. This together with Lemma 5.1 implies fixation.

Both parts of Theorem 1.2 directly follow from the previous lemma.

Proof of Theorem 1.2.a. Assume that (1.3) holds and that

$$
S(\Gamma, \tau)=\sum_{k>0}\left((k-2) \sum_{s:\lceil s / \tau\rceil=k} N(\Gamma, s)\right)>0 .
$$

Then, the expected weight becomes

$$
\begin{aligned}
E\left(W_{e}\right) & =\sum_{i, j \in V} \rho_{i} \rho_{j} \sum_{k>0}\left((k-2) \sum_{s:\lceil s / \tau\rceil=k} \mathbf{1}\{d(i, j)=s\}\right) \\
& =(1 / F)^{2} \sum_{k>0}\left((k-2) \sum_{s:\lceil s / \tau\rceil=k} \sum_{i, j \in V} \mathbf{1}\{d(i, j)=s\}\right) \\
& =(1 / F)^{2} \sum_{k>0}\left((k-2) \sum_{s:\lceil s / \tau\rceil=k} \operatorname{card}\{(i, j) \in V \times V: d(i, j)=s\}\right) \\
& =(1 / F)^{2} \sum_{k>0}\left((k-2) \sum_{s:\lceil s / \tau\rceil=k} N(\Gamma, s)\right) \\
& =(1 / F)^{2} S(\Gamma, \tau)>0
\end{aligned}
$$

which, according to Lemma 7.2, implies fixation.

Proof of Theorem 1.2.b. Assume that $\mathbf{d}>2 \tau$ and fix a pair $\left(i_{-}, i_{+}\right)$of antipodal vertices. Then, let $X, Y \geq 0$ such that

$$
2 X+(F-2) Y=1
$$

and assume that

$$
\rho_{i_{-}}=\rho_{i_{+}}=X \quad \text { and } \quad \rho_{i}=Y \quad \text { for all } \quad i \notin B:=\left\{i_{-}, i_{+}\right\} .
$$


To simplify the notation, we also introduce

$$
Q(i, j):=\sum_{k>0}\left((k-2) \sum_{s:\lceil s / \tau\rceil=k} \mathbf{1}\{d(i, j)=s\}\right)
$$

for all $(i, j) \in V \times V$. Then, the expected weight becomes

$$
\begin{aligned}
P(X, Y)= & \sum_{i, j \in V} \rho_{i} \rho_{j} Q(i, j) \\
= & \sum_{i, j \in B} \rho_{i} \rho_{j} Q(i, j)+\sum_{i \notin B} 2 \rho_{i} \rho_{i_{-}} Q\left(i, i_{-}\right) \\
\quad & \quad+\sum_{i \notin B} 2 \rho_{i} \rho_{i_{+}} Q\left(i, i_{+}\right)+\sum_{i, j \notin B} \rho_{i} \rho_{j} Q(i, j) \\
= & 2 Q\left(i_{-}, i_{+}\right) X^{2}+2\left(\sum_{i \notin B} Q\left(i, i_{-}\right)\right. \\
\quad & \left.\quad Q\left(i, i_{+}\right)\right) X Y+\sum_{i, j \notin B} Q(i, j) Y^{2} .
\end{aligned}
$$

This shows that $P$ is continuous in both $X$ and $Y$ and that

$$
\begin{aligned}
P(1 / 2,0) & =(1 / 2) Q\left(i_{-}, i_{+}\right) \\
& =(1 / 2) \sum_{k>0}\left((k-2) \sum_{s:\lceil s / \tau\rceil=k} \mathbf{1}\left\{d\left(i_{-}, i_{+}\right)=s\right\}\right) \\
& \geq(1 / 2)(3-2) \sum_{s>2 \tau} \mathbf{1}\left\{d\left(i_{-}, i_{+}\right)=s\right\}=1 / 2>0 .
\end{aligned}
$$

Therefore, according to Lemma 7.2, there is fixation of the process starting from any product measure whose densities are in some neighborhood of

$$
\rho_{i_{-}}=\rho_{i_{+}}=1 / 2 \quad \text { and } \quad \rho_{i}=0 \quad \text { for all } i \notin\left\{i_{-}, i_{+}\right\} .
$$

This proves the second part of Theorem 1.2.

\section{Proof of Theorem 1.3 (distance-regular graphs)}

To explain the intuition behind the proof of Theorem 1.3, recall that, when an active pile of size $s_{-}$jumps to the right onto a frozen pile of size $s_{+}$at edge $e$, the size of the latter pile becomes

$$
\xi_{t}(e)=d\left(\eta_{t}(e-1 / 2), \eta_{t}(e+1 / 2)\right)=d\left(\eta_{t-}(e-3 / 2), \eta_{t-}(e+1 / 2)\right)
$$

and the triangle inequality implies that

$$
s_{+}-s_{-}=\xi_{t-}(e)-\xi_{t-}(e-1) \leq \xi_{t}(e) \leq \xi_{t-}(e)+\xi_{t-}(e-1)=s_{+}+s_{-} .
$$

The exact distribution of the new size cannot be deduced in general from the size of the intersecting piles, indicating that the system of piles is not Markov. The key to the proof is that, at least when the underlying opinion graph is distance-regular, the system of piles becomes Markov. The first step is to show that, for all opinion graphs, the opinions on the left and on the right of a pile of size $s$ are conditioned to be at distance $s$ of each other but are otherwise independent, which follows from the fact that both opinions originate from two different ancestors at time zero, and the fact that the initial distribution is a product measure. If in addition the opinion graph is distance-regular then the number of possible opinions on the left and on the right of the pile, which is also the number of pairs of opinions at distance $s$ of each other, does not depend on the actual opinion on the left of the pile. This implies that, at least in theory, the new size distribution of a pile right after a collision can be computed explicitly. This is then used to prove that a jump of an active pile onto a pile of order $n>1$ reduces its order with probability at most

$$
p_{n}=\max \left\{\sum_{s:\lceil s / \tau\rceil=n-1} f\left(s_{-}, s_{+}, s\right) / h\left(s_{+}\right):\left\lceil s_{-} / \tau\right\rceil=1 \text { and }\left\lceil s_{+} / \tau\right\rceil=n\right\}
$$


while it increases its order with probability at least

$$
q_{n}=\min \left\{\sum_{s:\lceil s / \tau\rceil=n+1} f\left(s_{-}, s_{+}, s\right) / h\left(s_{+}\right):\left\lceil s_{-} / \tau\right\rceil=1 \text { and }\left\lceil s_{+} / \tau\right\rceil=n\right\} .
$$

In particular, the number of active piles that need to be sacrificed to turn a frozen pile into an active pile is stochastically larger than the hitting time to state 1 of a certain discrete-time birth and death process. To turn this heuristics into a proof, we let $x=e-1 / 2$ and

$$
x-1 \rightarrow_{t} x:=\text { the event that there is an arrow } x-1 \rightarrow x \text { at time } t .
$$

Then, we have the following lemma.

Lemma 8.1. Assume (1.3) and (1.7). For all $s \geq 0, s_{-} \leq \tau$ and $s_{+}>0$,

$$
\begin{aligned}
P\left(\xi_{t}(e)=s \mid\left(\xi_{t-}(e-1), \xi_{t-}(e)\right)=\left(s_{-}, s_{+}\right)\right. & \\
& \text {and } \left.x-1 \rightarrow_{t} x\right)=f\left(s_{-}, s_{+}, s\right) / h\left(s_{+}\right) .
\end{aligned}
$$

Proof: The first step is similar to the proof of Lanchier and Scarlatos (2013, Lemma 3). Due to one-dimensional nearest neighbor interactions, active paths cannot cross each other. In particular, the opinion dynamics preserve the ordering of the ancestral lineages therefore

$$
a(x-1, t-) \leq a(x, t-) \leq a(x+1, t-)
$$

where $a(z, t-)$ refers to the ancestor of $(z, t-)$, i.e., the unique source at time zero of an active path reaching $(z, t-)$. Since in addition $s_{-}, s_{+}>0$, given the conditioning in the statement of the lemma, the individuals at $x$ and $x \pm 1$ disagree at time $t-$ and so have different ancestors. This together with (8.2) implies that

$$
a(x-1, t-)<a(x, t-)<a(x+1, t-) .
$$

Now, we fix $i_{-}, j \in V$ such that $d\left(i_{-}, j\right)=s_{-}$and let

$$
B_{t-}\left(i_{-}, j\right):=\left\{\eta_{t-}(x-1)=i_{-} \text {and } \eta_{t-}(x)=j\right\} .
$$

Then, given this event and the conditioning in the statement of the lemma, the probability that the pile of particles at $e$ becomes of size $s$ is equal to

$$
\begin{gathered}
P\left(\xi_{t}(e)=s \mid B_{t-}\left(i_{-}, j\right) \text { and } \xi_{t-}(e)=s_{+} \text {and } x-1 \rightarrow_{t} x\right) \\
=P\left(d\left(i_{-}, \eta_{t-}(x+1)\right)=s \mid B\left(i_{-}, j\right)\right. \text { and } \\
\left.d\left(j, \eta_{t-}(x+1)\right)=s_{+} \text {and } x-1 \rightarrow_{t} x\right) \\
=\operatorname{card}\left\{i_{+}: d\left(i_{-}, i_{+}\right)=s \text { and } d\left(i_{+}, j\right)=s_{+}\right\} \\
\quad \div \operatorname{card}\left\{i_{+}: d\left(i_{+}, j\right)=s_{+}\right\}
\end{gathered}
$$

where the last equality follows from (1.3) and (8.3) which, together, imply that the opinion at $x+1$ just before the jump is independent of the other opinions on its left and chosen uniformly at random from the set of opinions at distance $s_{+}$of opinion $j$. Assuming in addition that the underlying opinion graph is a distanceregular graph (1.7), we also have

$$
\begin{aligned}
& \operatorname{card}\left\{i_{+}: d\left(i_{-}, i_{+}\right)=s \text { and } d\left(i_{+}, j\right)=s_{+}\right\} \\
& \quad=N\left(\Gamma,\left(i_{-}, s\right),\left(j, s_{+}\right)\right)=f\left(s_{-}, s_{+}, s\right) \\
& \operatorname{card}\left\{i_{+}: d\left(i_{+}, j\right)=s_{+}\right\} \\
& =N\left(\Gamma,\left(j, s_{+}\right)\right)=h\left(s_{+}\right) .
\end{aligned}
$$


In particular, the conditional probability in (8.4) does not depend on the particular choice of the pair of opinions $i_{-}$and $j$ from which it follows that

$$
\begin{aligned}
P\left(\xi_{t}(e)\right. & \left.=s \mid \xi_{t-}(e-1)=s_{-} \text {and } \xi_{t-}(e)=s_{+} \text {and } x-1 \rightarrow_{t} x\right) \\
& =P\left(\xi_{t}(e)=s \mid B_{t-}\left(i_{-}, j\right) \text { and } \xi_{t-}(e)=s_{+} \text {and } x-1 \rightarrow_{t} x\right)
\end{aligned}
$$

The lemma is then a direct consequence of (8.4)-(8.6).

As previously mentioned, it follows from Lemma 8.1 that, provided the opinion model starts from a product measure in which the density of each opinion is constant across space and the opinion graph is distance-regular, the system of piles itself is a Markov process. Another important consequence is the following lemma, which gives bounds for the probabilities that the jump of an active pile onto a frozen pile results in a reduction or an increase of its order.

Lemma 8.2. Let $x=e-1 / 2$. Assume (1.3) and (1.7). Then,

$$
\begin{aligned}
& P\left(\left\lceil\xi_{t}(e) / \tau\right\rceil<\left\lceil\xi_{t-}(e) / \tau\right\rceil \mid\right. \\
& \left.\left(\xi_{t-}(e-1), \xi_{t-}(e)\right)=\left(s_{-}, s_{+}\right) \text {and } x-1 \rightarrow_{t} x\right) \leq p_{n} \\
& P\left(\left\lceil\xi_{t}(e) / \tau\right\rceil>\left\lceil\xi_{t-}(e) / \tau\right\rceil \mid\right. \\
& \left.\left(\xi_{t-}(e-1), \xi_{t-}(e)\right)=\left(s_{-}, s_{+}\right) \text {and } x-1 \rightarrow_{t} x\right) \geq q_{n}
\end{aligned}
$$

whenever $0<\left\lceil s_{-} / \tau\right\rceil=1$ and $\left\lceil s_{+} / \tau\right\rceil=n>1$.

Proof: Let $p\left(s_{-}, s_{+}, s\right)$ be the conditional probability

$$
P\left(\xi_{t}(e)=s \mid\left(\xi_{t-}(e-1), \xi_{t-}(e)\right)=\left(s_{-}, s_{+}\right) \text {and } x-1 \rightarrow_{t} x\right)
$$

in the statement of Lemma 8.1. Then, the probability that the jump of an active pile onto the pile of order $n$ at edge $e$ results in a reduction of its order is

$$
\leq \max \left\{\sum_{s:\lceil s / \tau\rceil=n-1} p\left(s_{-}, s_{+}, s\right):\left\lceil s_{-} / \tau\right\rceil=1 \text { and }\left\lceil s_{+} / \tau\right\rceil=n\right\}
$$

while the probability that the jump of an active pile onto the pile of order $n$ at edge $e$ results in an increase of its order is

$$
\geq \min \left\{\sum_{s:\lceil s / \tau\rceil=n+1} p\left(s_{-}, s_{+}, s\right):\left\lceil s_{-} / \tau\right\rceil=1 \text { and }\left\lceil s_{+} / \tau\right\rceil=n\right\} .
$$

But according to Lemma 8.1, we have

$$
p\left(s_{-}, s_{+}, s\right)=f\left(s_{-}, s_{+}, s\right) / h\left(s_{+}\right)
$$

therefore (8.7)-(8.8) are equal to $p_{n}$ and $q_{n}$, respectively.

We refer to Figure 8.2 for a schematic illustration of the previous lemma. In order to prove the theorem, we now use Lemmas 8.1-8.2 to find a stochastic lower bound for the contribution of each edge. To express this lower bound, we let $X_{t}$ be the discrete-time birth and death Markov chain with transition probabilities

$$
p(n, n-1)=p_{n} \quad p(n, n)=1-p_{n}-q_{n} \quad p(n, n+1)=q_{n}
$$

for all $1<n<M:=\lceil\mathbf{d} / \tau\rceil$ and boundary conditions

$$
p(1,1)=1 \quad \text { and } \quad p(M, M-1)=1-p(M, M)=p_{M} .
$$

This process will allow us to retrace the history of a frozen pile until time $T_{e}$ when it becomes an active pile. To begin with, we use a first-step analysis to compute explicitly the expected value of the first hitting time to state 1. 
Lemma 8.3. Let $T_{n}:=\inf \left\{t: X_{t}=n\right\}$. Then,

$$
E\left(T_{1} \mid X_{0}=k\right)=1+\mathbf{W}(k) \text { for all } 0<k \leq M=\lceil\mathbf{d} / \tau\rceil .
$$

Proof: Let $\sigma_{n}:=E\left(T_{n-1} \mid X_{0}=n\right)$. Then, for all $1<n<M$,

$$
\begin{aligned}
\sigma_{n} & =p(n, n-1)+\left(1+\sigma_{n}\right) p(n, n)+\left(1+\sigma_{n}+\sigma_{n+1}\right) p(n, n+1) \\
& =p_{n}+\left(1+\sigma_{n}\right)\left(1-p_{n}-q_{n}\right)+\left(1+\sigma_{n}+\sigma_{n+1}\right) q_{n} \\
& =p_{n}+\left(1+\sigma_{n}\right)\left(1-p_{n}\right)+q_{n} \sigma_{n+1} \\
& =1+\left(1-p_{n}\right) \sigma_{n}+q_{n} \sigma_{n+1}
\end{aligned}
$$

from which it follows, using a simple induction, that

$$
\begin{aligned}
\sigma_{n}= & 1 / p_{n}+\sigma_{n+1} q_{n} / p_{n} \\
= & 1 / p_{n}+q_{n} /\left(p_{n} p_{n+1}\right)+\sigma_{n+2}\left(q_{n} q_{n+1}\right) /\left(p_{n} p_{n+1}\right) \\
= & \sum_{n \leq m<M}\left(q_{n} \cdots q_{m-1}\right) /\left(p_{n} \cdots p_{m}\right) \\
& \quad+\sigma_{M}\left(q_{n} \cdots q_{M-1}\right) /\left(p_{n} \cdots p_{M-1}\right) .
\end{aligned}
$$

Since $p(M, M-1)=1-p(M, M)=p_{M}$, we also have

$$
\sigma_{M}=E\left(T_{M-1} \mid X_{0}=M\right)=E\left(\operatorname{Geometric}\left(p_{M}\right)\right)=1 / p_{M} .
$$

Combining (8.9)-(8.10), we deduce that

$$
\sigma_{n}=\sum_{n \leq m \leq M}\left(q_{n} q_{n+1} \cdots q_{m-1}\right) /\left(p_{n} p_{n+1} \cdots p_{m}\right),
$$

which finally gives

$$
\begin{aligned}
E\left(T_{1} \mid X_{0}=k\right) & =\sum_{1<n \leq k} E\left(T_{n-1} \mid X_{0}=n\right)=\sum_{1<n \leq k} \sigma_{n} \\
& =\sum_{1<n \leq k} \sum_{n \leq m \leq M}\left(q_{n} \cdots q_{m-1}\right) /\left(p_{n} \cdots p_{m}\right)=1+\mathbf{W}(k) .
\end{aligned}
$$

This completes the proof.

The next lemma gives a lower bound for the contribution (5.6) of an edge $e$ that keeps track of the number of active piles that jump onto $e$ before the pile at $e$ becomes active. The key is to show how the number of jumps relates to the birth and death process. Before stating our next result, we recall that $T_{e}$ is the first time the pile of particles at edge $e$ becomes active.

Lemma 8.4. Assume (1.3) and (1.7). Then, for $1<k \leq\lceil\mathbf{d} / \tau\rceil$,

$$
E\left(\operatorname{cont}\left(e \mid T_{e}<\infty\right)\right) \geq \mathbf{W}(k) \text { when }\left\lceil\xi_{0}(e) / \tau\right\rceil=k .
$$

Proof: Since active piles have at most $\tau$ particles, the triangle inequality (8.1) implies that the jump of an active pile onto a frozen pile can only increase or decrease its size by at most $\tau$ particles, and therefore can only increase or decrease its order by at most one. In particular,

$$
P\left(\left|\left\lceil\xi_{t}(e) / \tau\right\rceil-\left\lceil\xi_{t-}(e) / \tau\right\rceil\right|>2 \mid x-1 \rightarrow_{t} x\right)=0 .
$$

This, together with the bounds in Lemma 8.2 and the fact that the outcomes of consecutive jumps of active piles onto a frozen pile are independent as explained in the proof of Lemma 8.1, implies that the order of a frozen pile before it becomes active dominates stochastically the state of the birth and death process $X_{t}$ before it reaches state 1 . In particular,

$$
E\left(\operatorname{cont}\left(e \mid T_{e}<\infty\right)\right) \geq-1+E\left(T_{1} \mid X_{0}=k\right) \quad \text { when } \quad\left\lceil\xi_{0}(e) / \tau\right\rceil=k .
$$



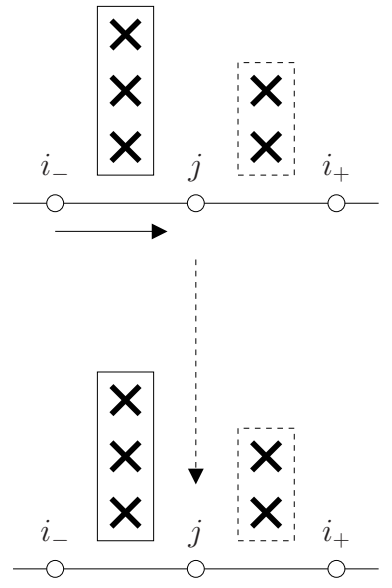

frozen pile (no update)
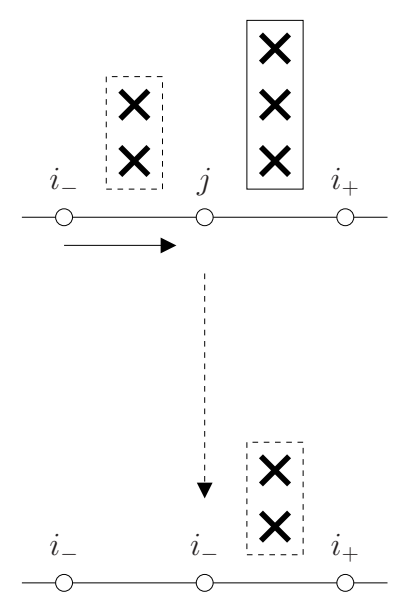

reduction of the order

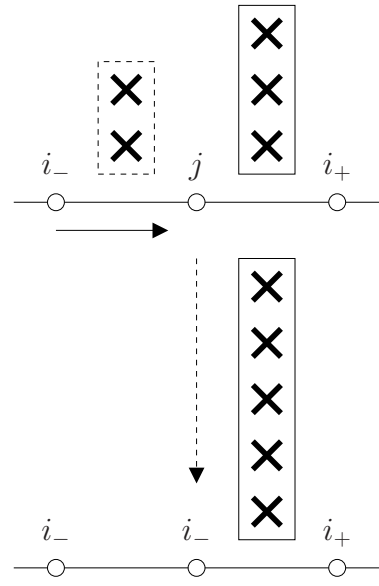

increase of the order

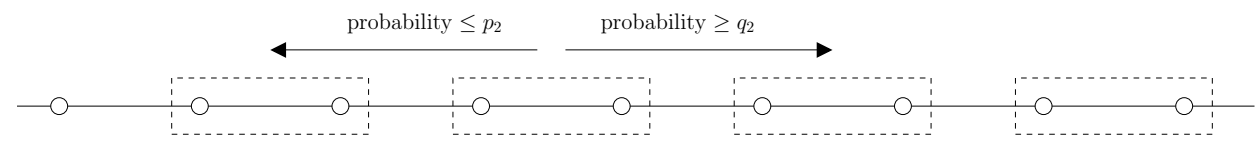

$\xi_{t}(e)=0 \quad\left\lceil\xi_{t}(e) / \tau\right\rceil=1 \quad\left\lceil\xi_{t}(e) / \tau\right\rceil=2 \quad\left\lceil\xi_{t}(e) / \tau\right\rceil=3 \quad\left\lceil\xi_{t}(e) / \tau\right\rceil=4$

FIGURE 8.2. Schematic illustration of the coupling between the opinion model and the system of piles along with their evolution rules. In our example, the threshold $\tau=2$, which makes piles with three or more particles frozen piles and piles with one or two particles active piles.

Using Lemma 8.3, we conclude that

$$
E\left(\operatorname{cont}\left(e \mid T_{e}<\infty\right)\right) \geq-1+(1+\mathbf{W}(k))=\mathbf{W}(k)
$$

whenever $\left\lceil\xi_{0}(e) / \tau\right\rceil=k$.

We now have all the tools to prove the theorem. The idea is the same as in the proof of Lemma 7.2 but relies on the previous lemma in place of Lemma 7.1.

Proof of Theorem 1.3. Assume (1.3) and (1.7) and

$$
S_{\text {reg }}(\Gamma, \tau)=\sum_{k>0}\left(\mathbf{W}(k) \sum_{s:\lceil s / \tau]=k} h(s)\right)>0 .
$$

Since the opinion graph is distance-regular,

$$
\begin{aligned}
P\left(\xi_{0}(e)=s\right) & =\sum_{i \in V} P\left(\xi_{0}(e)=s \mid \eta_{0}(e-1 / 2)=i\right) P\left(\eta_{0}(e-1 / 2)=i\right) \\
& =\sum_{i \in V} F^{-1} \operatorname{card}\{j \in V: d(i, j)=s\} P\left(\eta_{0}(e-1 / 2)=i\right) \\
& =\sum_{i \in V} F^{-1} h(s) P\left(\eta_{0}(e-1 / 2)=i\right)=F^{-1} h(s) .
\end{aligned}
$$


Using also Lemma 8.4, we get

$$
\begin{aligned}
E\left(\operatorname{cont}\left(e \mid T_{e}<\infty\right)\right) & \geq \sum_{k>0} \mathbf{W}(k) P\left(\left\lceil\xi_{0}(e) / \tau\right\rceil=k\right) \\
& =\sum_{k>0} \mathbf{W}(k) P\left((k-1) \tau<\xi_{0}(e) \leq k \tau\right) \\
& =\sum_{k>0} \mathbf{W}(k) \sum_{s:\lceil s / \tau\rceil=k} F^{-1} h(s) \\
& =F^{-1} S_{\mathrm{reg}}(\Gamma, \tau)>0 .
\end{aligned}
$$

Now, let $\mathbf{W}_{e}$ be the collection of random variables

$$
\mathbf{W}_{e}:=\sum_{k>0} \mathbf{W}(k) \mathbf{1}\left\{\xi_{0}(e)=k\right\} \quad \text { for all } \quad e \in \mathbb{Z}+1 / 2 \text {. }
$$

Using Lemma 6.1 and the fact the number of collisions to turn a frozen pile into an active pile is independent for different frozen piles, we deduce that there exists a constant $c_{2}>0$ such that

$$
\begin{aligned}
& P\left(\sum_{e \in(0, N)} \operatorname{cont}\left(e \mid T_{e}<\infty\right) \leq 0\right) \leq P\left(\sum_{e \in(0, N)} \mathbf{W}_{e} \leq 0\right) \\
& =P\left(\sum_{e \in(0, N)}\left(\mathbf{W}_{e}-E\left(\mathbf{W}_{e}\right)\right) \notin(-\epsilon N, \epsilon N)\right) \leq \exp \left(-c_{2} N\right)
\end{aligned}
$$

for all $N$ large. This, together with (5.7), implies that

$$
\begin{aligned}
P\left(H_{N}\right) & \leq P\left(\sum_{e \in(l, r)} \operatorname{cont}\left(e \mid T_{e}<\infty\right) \leq 0 \text { for some } l<-N \text { and } r \geq 0\right) \\
& \leq \sum_{l<-N} \sum_{r \geq 0} \exp \left(-c_{2}(r-l)\right) \rightarrow 0
\end{aligned}
$$

as $N \rightarrow \infty$. In particular, fixation follows from Lemma 5.1.

\section{Proof of Corollaries 2.1-2.5}

This section is devoted to the proof of Corollaries 2.1-2.5 that give sufficient conditions for fluctuation and fixation for the opinion graphs shown in Figure 2.1. To begin with, we prove the fluctuation part of all the corollaries at once.

Proof of Corollaries 2.1-2.5 (fluctuation). We start with the tetrahedron. In this case, the diameter equals one therefore, whenever the threshold is positive, the system reduces to a four-opinion voter model, which is known to fluctuate according to Arratia (1983). To deal with paths and stars, we recall that combining Theorem 1.1.a and Lemma 4.1 gives fluctuation when $\mathbf{r} \leq \tau$. Recalling also the expression of the radius from Table 2.1 implies fluctuation when

$$
\begin{aligned}
F \leq 2 \tau+1 & \text { for the path with } F \text { vertices } \\
r \leq \tau & \text { for the star with } b \text { branches of length } r .
\end{aligned}
$$

For the other graphs, it suffices to find a partition that satisfies (1.5). For the remaining four regular polyhedra and the hypercubes, we observe that there is a unique vertex at distance $\mathbf{d}$ of any given vertex. In particular, fixing an arbitrary vertex $i_{-}$and setting

$$
V_{1}:=\left\{i_{-}, i_{+}\right\} \quad \text { and } \quad V_{2}:=V \backslash V_{1}
$$

where $i_{-}$and $i_{+}$are antipodal defines a partition of the set of opinions such that

$$
d(i, j) \leq \mathbf{d}-1 \quad \text { for all } \quad(i, j) \in V_{1} \times V_{2} .
$$

Recalling the expression of the diameter from Table 2.1 and using Theorem 1.1.a give the fluctuation parts of Corollaries 2.3 and 2.5. Using the exact same approach implies fluctuation when the opinion graph is a cycle with an even number of vertices 
and $F \leq 2 \tau+2$. For cycles with an odd number of vertices, we again use Lemma 4.1 to deduce fluctuation if

$$
\lfloor F / 2\rfloor=\mathbf{r} \leq \tau \quad \text { if and only if } \quad F \leq 2 \tau+1 \quad \text { if and only if } \quad F \leq 2 \tau+2 \text {, }
$$

where the last equivalence is true because $F$ is odd.

We now prove the fixation part of the corollaries. Depending on the opinion graphs, the proofs rely on Theorem 1.2 or Theorem 1.3. The first two classes of graphs, paths and stars, are not distance-regular therefore, to study the behavior of the model for these opinion graphs, we rely on the first part of Theorem 1.2.

Proof of Corollary 2.1 (path). Assume that $4 \tau<\mathbf{d}=F-1 \leq 5 \tau$. Then,

$$
\begin{aligned}
S(\Gamma, \tau)= & \sum_{k>0}\left((k-2) \sum_{s:\lceil s / \tau\rceil=k} N(\Gamma, s)\right) \\
= & \sum_{0<k \leq 4}\left((k-2) \sum_{s:\lceil s / \tau\rceil=k} 2(F-s)\right)+3 \sum_{4 \tau<s \leq d} 2(F-s) \\
= & \sum_{0<k \leq 4}((k-2)(2 F \tau-(k \tau)(k \tau+1)+((k-1) \tau)((k-1) \tau+1)) \\
& \quad+3(2 F(F-4 \tau-1)-F(F-1)+4 \tau(4 \tau+1)) \\
= & 4 F \tau+\tau(\tau+1)+2 \tau(2 \tau+1)+3 \tau(3 \tau+1) \\
\quad & \quad+4 \tau(4 \tau+1)+6 F(F-4 \tau-1)-3 F(F-1) \\
= & 3 F^{2}-(20 \tau+3) F+10(3 \tau+1) \tau .
\end{aligned}
$$

Since the largest root $F_{+}(\tau)$ of this polynomial satisfies

$$
4 \tau \leq F_{+}(\tau)-1=(1 / 6)\left(20 \tau+3+\sqrt{40 \tau^{2}+9}\right)-1 \leq 5 \tau \quad \text { for all } \quad \tau \geq 1
$$

and since for each $\tau$ the function $F \mapsto S(\Gamma, \tau)$ is nondecreasing, we deduce that fixation occurs under the assumptions of the lemma according to Theorem 1.2.

The case of the star with $b$ branches of equal length $r$ is more difficult mainly because there are two different expressions for the number of pairs of vertices at a given distance of each other depending on whether the distance is smaller or larger than the branches' length. In the next lemma, we compute the number of pairs of vertices at a given distance of each other, which we then use to find a condition for fixation when the opinion graph is a star.

Lemma 9.1. For the star with $b$ branches of length $r$,

$$
\begin{aligned}
& N(\Gamma, s)=b(2 r+(b-3)(s-1)) \quad \text { for all } s \in(0, r] \\
& =b(b-1)(2 r-s+1) \quad \text { for all } \quad s \in(r, 2 r] \text {. }
\end{aligned}
$$

Proof: Let $n_{1}(s)$ and $n_{2}(s)$ be respectively the number of directed paths of length $s$ embedded in a given branch of the star and the total number of directed paths of length $s$ embedded in a given pair of branches of the star. Then, as in the proof of the corollary for paths,

$$
n_{1}(s)=2(r+1-s) \quad \text { and } \quad n_{2}(s)=2(2 r+1-s) \quad \text { for all } \quad s \leq r .
$$


Since there are $b$ branches and $(1 / 2)(b-1) b$ pairs of branches, and since self-avoiding paths embedded in the star cannot intersect more than two branches,

$$
\begin{aligned}
N(\Gamma, s) & =b n_{1}(s)+((1 / 2)(b-1) b)\left(n_{2}(s)-2 n_{1}(s)\right) \\
& =2 b(r+1-s)+b(b-1)(s-1) \\
& =b(2 r+2(1-s)+(b-1)(s-1))=b(2 r+(b-3)(s-1))
\end{aligned}
$$

for all $s \leq r$. To deal with the case $s>r$, we let $o$ be the center of the star and observe that there is no vertex at distance $s$ of vertices which are close to the center whereas there are $b-1$ vertices at distance $s$ from vertices which are far from the center. More precisely,

$$
\begin{array}{lll}
\operatorname{card}\{j \in V: d(i, j)=s\}=0 & \text { when } & d(i, o)<s-r \\
\operatorname{card}\{j \in V: d(i, j)=s\}=b-1 & \text { when } & d(i, o) \geq s-r .
\end{array}
$$

The number of directed paths of length $s$ is then given by

$$
\begin{aligned}
N(\Gamma, s) & =(b-1) \operatorname{card}\{i \in V: d(i, o) \geq s-r\} \\
& =b(b-1)(r-(s-r-1))=b(b-1)(2 r-s+1)
\end{aligned}
$$

for all $s>r$. This completes the proof of the lemma.

Proof of Corollary 2.2 (star). Assume that $3 \tau<\mathbf{d}=2 r \leq 4 \tau$. Then,

$$
\begin{aligned}
S(\Gamma, \tau) & =\sum_{k>0}\left((k-2) \sum_{s:\lceil s / \tau\rceil=k} N(\Gamma, s)\right) \\
& =-\sum_{0<s \leq \tau} N(\Gamma, s)+\sum_{2 \tau<s \leq 3 \tau} N(\Gamma, s)+2 \sum_{3 \tau<s \leq 2 r} N(\Gamma, s) .
\end{aligned}
$$

Since $\tau<r \leq 2 \tau$, it follows from Lemma 9.1 that

$$
\begin{aligned}
S(\Gamma, \tau)= & -\sum_{0<s \leq \tau} b(2 r+(b-3)(s-1)) \\
& +\sum_{2 \tau<s \leq 3 \tau} b(b-1)(2 r-s+1)+2 \sum_{3 \tau<s \leq 2 r} b(b-1)(2 r-s+1) \\
= & -b(2 r-b+3) \tau-(b / 2)(b-3) \tau(\tau+1) \\
& +b(b-1)(2 r+1) \tau+(b / 2)(b-1)(2 \tau(2 \tau+1)-3 \tau(3 \tau+1)) \\
& +2 b(b-1)(2 r+1)(2 r-3 \tau)+b(b-1)(3 \tau(3 \tau+1)-2 r(2 r+1)) .
\end{aligned}
$$

Expanding and simplifying, we get

$$
(1 / b) S(\Gamma, \tau)=4(b-1) r^{2}+2((4-5 b) \tau+b-1) r+(6 b-5) \tau^{2}+(1-2 b) \tau .
$$

As for paths, the result is a direct consequence of Theorem 1.2.

The remaining graphs in Figure 2.1 are distance-regular, which makes Theorem 1.3 applicable. Note that the conditions for fixation in the last three corollaries give minimal values for the confidence threshold that lie between one third and one half of the diameter. In particular, we apply the theorem in the special case when $\lceil\mathbf{d} / \tau\rceil=3$. In this case, we have

$$
\mathbf{W}(1)=-1 \quad \mathbf{W}(2)=\mathbf{W}(1)+\left(1 / p_{2}\right)\left(1+q_{2} / p_{3}\right) \quad \mathbf{W}(3)=\mathbf{W}+1 / p_{3}
$$


so the left-hand side of (1.8) becomes

$$
\begin{aligned}
S_{\mathrm{reg}}(\Gamma, \tau)= & \sum_{0<k \leq 3}\left(\mathbf{W}(k) \sum_{s:\lceil s / \tau\rceil=k} h(s)\right) \\
= & -(h(1)+h(2)+\cdots+h(\mathbf{d})) \\
& +\left(1 / p_{2}\right)\left(1+q_{2} / p_{3}\right)(h(\tau+1)+h(\tau+2)+\cdots+h(\mathbf{d})) \\
& +\left(1 / p_{3}\right)(h(2 \tau+1)+h(2 \tau+2)+\cdots+h(\mathbf{d})) .
\end{aligned}
$$

This expression is used repeatedly to prove the remaining corollaries.

Proof of Corollary 2.3 (cube). When $\Gamma$ is the cube and $\tau=1$, we have

$$
p_{2}=f(1,2,1) / h(2)=2 / 3 \quad \text { and } \quad q_{2}=f(1,2,3) / h(2)=1 / 3
$$

which, together with (9.1) and the fact that $p_{3} \leq 1$, implies that

$$
\begin{aligned}
S_{\mathrm{reg}}(\Gamma, 1) & \geq-(h(1)+h(2)+h(3))+\left(1 / p_{2}\right)\left(1+q_{2}\right)(h(2)+h(3))+h(3) \\
& =-(3+3+1)+(3 / 2)(1+1 / 3)(3+1)+1=2>0 .
\end{aligned}
$$

This proves fixation according to Theorem 1.3.

Proof of Corollary 2.3 (icosahedron). When $\Gamma$ is the icosahedron and $\tau=1$,

$$
p_{2}=f(1,2,1) / h(2)=2 / 5 \quad \text { and } \quad q_{2}=f(1,2,3) / h(2)=1 / 5 .
$$

Using in addition (9.1) and the fact that $p_{3} \leq 1$, we obtain

$$
\begin{aligned}
S_{\mathrm{reg}}(\Gamma, 1) & \geq-(h(1)+h(2)+h(3))+\left(1 / p_{2}\right)\left(1+q_{2}\right)(h(2)+h(3))+h(3) \\
& =-(5+5+1)+(5 / 2)(1+1 / 5)(5+1)+1=8>0
\end{aligned}
$$

which, according to Theorem 1.3, implies fixation.

Proof of Corollary 2.3 (dodecahedron). Fixation of the opinion model when the threshold equals one directly follows from Theorem 1.2 since in this case

$$
\begin{aligned}
F^{-1} S(\Gamma, 1) & =(1 / 20)(-h(1)+h(3)+2 h(4)+3 h(5)) \\
& =(1 / 20)(-3+6+2 \times 3+3 \times 1)=3 / 5>0 .
\end{aligned}
$$

However, when the threshold $\tau=2$,

$$
\begin{aligned}
F^{-1} S(\Gamma, 2) & =(1 / 20)(-h(1)-h(2)+h(5)) \\
& =(1 / 20)(-3-6+1)=-2 / 5<0
\end{aligned}
$$

so we use Theorem 1.3 instead: when $\tau=2$, we have

$$
\begin{aligned}
p_{2} & =\max \left\{\sum_{s=1,2} f\left(s_{-}, s_{+}, s\right) / h\left(s_{+}\right): s_{-}=1,2 \text { and } s_{+}=3,4\right\} \\
& =\max \{f(1,3,2) / h(3),(f(2,3,2)+f(2,3,1)) / h(3), f(2,4,2) / h(4)\} \\
& =\max \{2 / 6,(2+1) / 6,1 / 3\}=1 / 2 .
\end{aligned}
$$

In particular, using (9.1) and the fact that $p_{3} \leq 1$ and $q_{2} \geq 0$, we get

$$
\begin{aligned}
S_{\mathrm{reg}}(\Gamma, 2) \geq & -(h(1)+h(2)+h(3)+h(4)+h(5)) \\
& \quad+\left(1 / p_{2}\right)(h(3)+h(4)+h(5))+h(5) \\
= & -(3+6+6+3+1)+2 \times(6+3+1)+1=2>0,
\end{aligned}
$$


which again gives fixation.

Proof of Corollary 2.4 (cycle). Regardless of the parity of $F$,

$$
\begin{aligned}
& f\left(s_{-}, s_{+}, s\right)=0 \quad \text { when } \quad s_{-} \leq s_{+} \leq \mathbf{d} \quad \text { and } \quad s>s_{+}-s_{-} \\
& f\left(s_{-}, s_{+}, s\right)=1 \quad \text { when } \quad s_{-} \leq s_{+} \leq \mathbf{d} \quad \text { and } \quad s=s_{+}-s_{-}
\end{aligned}
$$

while the number of vertices at distance $s_{+}$of a given vertex is

$$
h\left(s_{+}\right)=2 \text { for all } s_{+}<F / 2 \text { and } h\left(s_{+}\right)=1 \text { when } s_{+}=F / 2 \in \mathbb{N} .
$$

Assume that $F=4 \tau+2$. Then, $\mathbf{d}=2 \tau+1$ so it follows from (9.2)-(9.3) that

$$
\begin{aligned}
p_{2} & =\max \left\{\sum_{s:\lceil s / \tau\rceil=1} f\left(s_{-}, s_{+}, s\right) / h\left(s_{+}\right):\left\lceil s_{-} / \tau\right\rceil=1 \text { and }\left\lceil s_{+} / \tau\right\rceil=2\right\} \\
& =\max \left\{f\left(s_{-}, s_{+}, s_{+}-s_{-}\right) / h\left(s_{+}\right):\left\lceil s_{-} / \tau\right\rceil=1 \text { and }\left\lceil s_{+} / \tau\right\rceil=2\right\} \\
& =\max \left\{f\left(s_{-}, s_{+}, s_{+}-s_{-}\right) / h\left(s_{+}\right):\left\lceil s_{+} / \tau\right\rceil=2\right\}=1 / 2 .
\end{aligned}
$$

Using in addition that $p_{3} \leq 1$ and $q_{2} \geq 0$ together with (9.1), we get

$$
\begin{aligned}
S_{\text {reg }}(\Gamma, \tau) \geq & -(h(1)+h(2)+\cdots+h(2 \tau+1)) \\
& +\left(1 / p_{2}\right)(h(\tau+1)+h(\tau+2)+\cdots+h(2 \tau+1))+h(2 \tau+1) \\
= & -(4 \tau+1)+2 \times(2 \tau+1)+1=2>0 .
\end{aligned}
$$

In particular, the corollary follows from Theorem 1.3.

Proof of corollary 2.5 (hypercube). The first part of the corollary has been explained heuristically in Adamopoulos and Scarlatos (2012). To turn it into a proof, we first observe that opinions on the hypercube can be represented by vectors with coordinates equal to zero or one while the distance between two opinions is the number of coordinates the two corresponding vectors disagree on. In particular, the number of opinions at distance $s$ of a given opinion, namely $h(s)$, is equal to the number of subsets of size $s$ of a set of size $d$, therefore

$$
h(s)=\left(\begin{array}{l}
d \\
s
\end{array}\right)=\left(\begin{array}{c}
d \\
d-s
\end{array}\right)=h(d-s) \quad \text { for } \quad s=0,1, \ldots, d .
$$

It follows that, for $d=3 \tau+1$,

$$
\begin{aligned}
2^{-d} S(\Gamma, \tau) & =-h(1)-\cdots-h(\tau)+h(2 \tau+1)+\cdots+h(d-1)+2 h(d) \\
& =h(d-1)-h(1)+\cdots+h(d-\tau)-h(\tau)+2 h(d) \\
& =2 h(d)=2>0 .
\end{aligned}
$$

Since in addition $d \mapsto S(\Gamma, \tau)$ is nondecreasing, a direct application of Theorem 1.2 gives the first part of the corollary. The second part is more difficult. Note that, to prove this part, it suffices to show that, for each $\sigma>0$, fixation occurs when

$$
d=(2+3 \sigma) \tau \text { and } \tau \text { is large. }
$$

The main difficulty is to find a good upper bound for $p_{2}$. This relies on properties of the hypergeometric random variable. Let $u$ and $v$ be two opinions at distance $s_{-}$ of each other. By symmetry, we may assume without loss of generality that both vectors disagree on their first $s_{-}$coordinates. Then, changing each of the first $s_{-}$ coordinates in either one vector or the other vector and changing each of the remaining coordinates in either both vectors simultaneously or none of the vectors 
result in the same vector. In particular, choosing a vector $w$ such that

$$
d(u, w)=s_{+} \quad \text { and } \quad d(v, w)=s
$$

is equivalent to choosing $a$ of the first $s_{-}$coordinates and then choosing $b$ of the remaining $d-s_{-}$coordinates with the following constraint:

$$
a+b=s_{+} \quad \text { and } \quad\left(s_{-}-a\right)+b=s .
$$

In particular, letting $K:=\left\lceil(1 / 2)\left(s_{-}+s_{+}-\tau\right)\right\rceil$, we have

$$
\sum_{s=1}^{\tau} f\left(s_{-}, s_{+}, s\right)=\sum_{a=K}^{s_{-}}\left(\begin{array}{c}
s_{-} \\
a
\end{array}\right)\left(\begin{array}{l}
d-s_{-} \\
s_{+}-a
\end{array}\right)=h\left(s_{+}\right) P(Z \geq K)
$$

where $Z=$ Hypergeometric $\left(d, s_{-}, s_{+}\right)$. In order to find an upper bound for the probability $p_{2}$ and deduce fixation, we first prove the following lemma about the hypergeometric random variable.

Lemma 9.2. Assume (9.5), that $\left\lceil s_{-} / \tau\right\rceil=1$ and $\left\lceil s_{+} / \tau\right\rceil=2$. Then,

$$
P(Z \geq K)=\sum_{a=K}^{s_{-}}\left(\begin{array}{c}
s_{-} \\
a
\end{array}\right)\left(\begin{array}{c}
d-s_{-} \\
s_{+}-a
\end{array}\right)\left(\begin{array}{c}
d \\
s_{+}
\end{array}\right)^{-1} \leq 1 / 2 .
$$

Proof: The proof is made challenging by the fact that there is no explicit expression for the cumulative distribution function of the hypergeometric random variable and the idea is to use a combination of symmetry arguments and large deviation estimates. Symmetry is used to prove the result when $s_{-}$is small while large deviation estimates are used for larger values. Note that the result is trivial when $s_{+}>s_{-}+\tau$ since in this case the sum in the statement of the lemma is empty so equal to zero. To prove the result when the sum is nonempty, we distinguish two cases.

Small active piles - Assume that $s_{-}<\sigma \tau$. Then,

$$
\begin{gathered}
s_{+} \leq s_{-}+\tau<(1+\sigma) \tau=(1 / 2)(d-\sigma \tau)<(1 / 2)\left(d-s_{-}\right) \\
K \geq(1 / 2)\left(s_{-}+s_{+}-\tau\right)>s_{-} / 2>s_{-}-K
\end{gathered}
$$

from which it follows that

$$
\left(\begin{array}{c}
s_{-} \\
a
\end{array}\right)\left(\begin{array}{c}
d-s_{-} \\
s_{+}-a
\end{array}\right) \leq\left(\begin{array}{c}
s_{-} \\
a
\end{array}\right)\left(\begin{array}{c}
d-s_{-} \\
s_{+}-s_{-}+a
\end{array}\right) \quad \text { for all } \quad K \leq a \leq s_{-} .
$$

Using (9.7) and again the second part of (9.6), we deduce that

$$
\begin{aligned}
h\left(s_{+}\right) P(Z \geq K) & =\sum_{a=K}^{s_{-}}\left(\begin{array}{c}
s_{-} \\
a
\end{array}\right)\left(\begin{array}{c}
d-s_{-} \\
s_{+}-a
\end{array}\right) \leq \sum_{a=K}^{s_{-}}\left(\begin{array}{c}
s_{-} \\
a
\end{array}\right)\left(\begin{array}{c}
d-s_{-} \\
s_{+}-s_{-}+a
\end{array}\right) \\
& =\sum_{a=0}^{s_{-} K}\left(\begin{array}{c}
s_{-} \\
s_{-}-a
\end{array}\right)\left(\begin{array}{c}
d-s_{-} \\
s_{+}-a
\end{array}\right) \leq \sum_{a=0}^{K-1}\left(\begin{array}{c}
s_{-} \\
a
\end{array}\right)\left(\begin{array}{c}
d-s_{-} \\
s_{+}-a
\end{array}\right) .
\end{aligned}
$$

In particular, we have $P(Z \geq K) \leq P(Z<K)$, which gives the result.

Larger active piles - Assume that $\sigma \tau \leq s_{-} \leq \tau$. In this case, the result is a consequence of the following large deviation estimates for the hypergeometric 
random variable:

$$
\begin{aligned}
P(Z & \left.\geq\left(\frac{s_{-}}{d}+\epsilon\right) s_{+}\right) \\
& \leq\left(\left(\frac{s_{-}}{s_{-}+\epsilon d}\right)^{s_{-} / d+\epsilon}\left(\frac{d-s_{-}}{d-s_{-}-\epsilon d}\right)^{1-s_{-} / d-\epsilon}\right)^{s_{+}}
\end{aligned}
$$

for all $0<\epsilon<1-s_{-} / d$, that can be found in Hoeffding (1963). Note that

$$
\begin{aligned}
d\left(s_{+}+s_{-}-\tau\right)-2 s_{+} s_{-} & =\left(d-2 s_{-}\right) s_{+}+d\left(s_{-}-\tau\right) \\
& \geq\left(d-2 s_{-}\right)(\tau+1)+d\left(s_{-}-\tau\right) \geq(d-2 \tau) s_{-} \\
& =3 \sigma \tau s_{-}=\left(3 \sigma \tau / 2 s_{+}\right)\left(2 s_{+} s_{-}\right) \geq(3 \sigma / 4)\left(2 s_{+} s_{-}\right)
\end{aligned}
$$

for all $\tau<s_{+} \leq 2 \tau$. It follows that

$$
\begin{aligned}
K \geq \frac{s_{+}+s_{-}-\tau}{2} & \geq\left(1+\frac{3 \sigma}{4}\right) \frac{s_{+} s_{-}}{d} \\
= & \left(\frac{s_{-}}{d}+\frac{3 \sigma s_{-}}{4 d}\right) s_{+} \geq\left(\frac{s_{-}}{d}+\frac{\sigma^{2}}{3}\right) s_{+}
\end{aligned}
$$

which, together with (9.8) for $\epsilon=\sigma^{2} / 3$, gives

$$
\begin{aligned}
P(Z \geq K) & \leq P\left(Z \geq\left(\frac{s_{-}}{d}+\epsilon\right) s_{+}\right) \\
& \leq\left(\frac{s_{-}}{s_{-}+\epsilon d}\right)^{s_{+} s_{-} / d} \leq\left(\frac{3 s_{-}}{3 s_{-}+\sigma^{2} d}\right)^{s_{+} s_{-} / d} \\
& \leq\left(\frac{3}{3+2 \sigma^{2}}\right)^{(\sigma / 3) s_{+}} \leq\left(\frac{3}{3+2 \sigma^{2}}\right)^{(\sigma / 3) \tau} .
\end{aligned}
$$

Since this tends to zero as $\tau \rightarrow \infty$, the proof is complete.

It directly follows from the lemma that

$$
p_{2}=\max \left\{\sum_{s:\lceil s / \tau\rceil=1} f\left(s_{-}, s_{+}, s\right) / h\left(s_{+}\right):\left\lceil s_{-} / \tau\right\rceil=1 \text { and }\left\lceil s_{+} / \tau\right\rceil=2\right\} \leq 1 / 2 .
$$

This, together with (9.1) and $p_{3} \leq 1$ and $q_{2} \geq 0$, implies that

$$
\begin{aligned}
S_{\text {reg }}(\Gamma, \tau) & \geq-h(1)-\cdots-h(d)+\left(1 / p_{2}\right) h(\tau+1)+\cdots+\left(1 / p_{2}\right) h(d) \\
& \geq-h(1)-\cdots-h(d)+2 h(\tau+1)+\cdots+2 h(d) \\
& =-h(1)-\cdots-h(\tau)+h(\tau+1)+\cdots+h(d) .
\end{aligned}
$$

Finally, using again (9.4) and the fact that $d>2 \tau$, we deduce that

$$
\begin{aligned}
S_{\mathrm{reg}}(\Gamma, \tau) & \geq-h(1)-\cdots-h(\tau)+h(\tau+1)+\cdots+h(d) \\
& \geq h(d-1)-h(1)+\cdots+h(d-\tau)-h(\tau)+h(d) \\
& =h(d)=1>0 .
\end{aligned}
$$

The corollary follows once more from Theorem 1.3.

Acknowledgment. The authors would like to thank an anonymous referee whose comments help improve the presentation of the results. Nicolas Lanchier was supported in part by NSF Grant DMS-10-05282 and NSA Grant MPS-14-040958, and 
Stylianos Scarlatos by an IKY Fellowship of Excellence for Postgraduate Studies in Greece - Siemens Program 2014-2015.

\section{References}

A. Adamopoulos and S. Scarlatos. Emulation and complementarity in onedimensional alternatives of the axelrod model with binary features. Complexity 17 (3), 43-49 (2012). DOI: 10.1002/cplx.20391.

R. Arratia. Site recurrence for annihilating random walks on $\mathbf{Z}_{d}$. Ann. Probab. 11 (3), 706-713 (1983). MR704557.

R. Axelrod. The dissemination of culture. J. of Conflict Resolut. 41 (2), 203-226 (1997). DOI: 10.1177/0022002797041002001.

M. A. Boudourides and S. Scarlatos. Choice polarization on a social influence network. Proceedings of Polarization and Conflict 2nd Annual Summer Meeting, PAC Research Group, Konstanz (2005).

M. Bramson and D. Griffeath. Flux and fixation in cyclic particle systems. Ann. Probab. 17 (1), 26-45 (1989). MR972768.

P. Clifford and A. Sudbury. A model for spatial conflict. Biometrika 60, 581-588 (1973). MR0343950.

G. Deffuant, D. Neau, F. Amblard and G. Weisbuch. Mixing beliefs among interacting agents. Adv. Compl. Sys. 3, 87-98 (2000). DOI: 10.1142/S0219525900000078.

T. E. Harris. Nearest-neighbor Markov interaction processes on multidimensional lattices. Advances in Math. 9, 66-89 (1972). MR0307392.

W. Hoeffding. Probability inequalities for sums of bounded random variables. J. Amer. Statist. Assoc. 58, 13-30 (1963). MR0144363.

R. A. Holley and T. M. Liggett. Ergodic theorems for weakly interacting infinite systems and the voter model. Ann. Probability 3 (4), 643-663 (1975). MR0402985.

Y. Itoh, C. Mallows and L. Shepp. Explicit sufficient invariants for an interacting particle system. J. Appl. Probab. 35 (3), 633-641 (1998). MR1659528.

N. Lanchier. The Axelrod model for the dissemination of culture revisited. Ann. Appl. Probab. 22 (2), 860-880 (2012). MR2953571.

N. Lanchier and P.-H. Moisson. Fixation results for the two-feature axelrod model with a variable number of opinions. J. Theoret. Probab. 29 (4), 1554-1580 (2016). MR3571254.

N. Lanchier and S. Scarlatos. Fixation in the one-dimensional Axelrod model. Ann. Appl. Probab. 23 (6), 2538-2559 (2013). MR3127944.

N. Lanchier and S. Scarlatos. Clustering and coexistence in the one-dimensional vectorial Deffuant model. ALEA Lat. Am. J. Probab. Math. Stat. 11 (1), 541-564 (2014). MR3274645.

N. Lanchier and J. Schweinsberg. Consensus in the two-state Axelrod model. Stochastic Process. Appl. 122 (11), 3701-3717 (2012). MR2965921.

S. Scarlatos. Voter models with confidence parameter. Ph.D. thesis, University of Patras, Greece (2013).

F. Vazquez, P. L. Krapivsky and S. Redner. Constrained opinion dynamics: freezing and slow evolution. J. Phys. A 36 (3), L61-L68 (2003). MR1959419. 